\title{
Nested Arrays: A Novel Approach to Array Processing With Enhanced Degrees of Freedom
}

\author{
Piya Pal, Student Member, IEEE, and P. P. Vaidyanathan, Fellow, IEEE
}

\begin{abstract}
A new array geometry, which is capable of significantly increasing the degrees of freedom of linear arrays, is proposed. This structure is obtained by systematically nesting two or more uniform linear arrays and can provide $O\left(N^{2}\right)$ degrees of freedom using only $N$ physical sensors when the second-order statistics of the received data is used. The concept of nesting is shown to be easily extensible to multiple stages and the structure of the optimally nested array is found analytically. It is possible to provide closed form expressions for the sensor locations and the exact degrees of freedom obtainable from the proposed array as a function of the total number of sensors. This cannot be done for existing classes of arrays like minimum redundancy arrays which have been used earlier for detecting more sources than the number of physical sensors. In minimum-input-minimum-output (MIMO) radar, the degrees of freedom are increased by constructing a longer virtual array through active sensing. The method proposed here, however, does not require active sensing and is capable of providing increased degrees of freedom in a completely passive setting. To utilize the degrees of freedom of the nested co-array, a novel spatial smoothing based approach to DOA estimation is also proposed, which does not require the inherent assumptions of the traditional techniques based on fourth-order cumulants or quasi stationary signals. As another potential application of the nested array, a new approach to beamforming based on a nonlinear preprocessing is also introduced, which can effectively utilize the degrees of freedom offered by the nested arrays. The usefulness of all the proposed methods is verified through extensive computer simulations.
\end{abstract}

Index Terms-Beamforming, co-array, direction-of-arrival (DOA) estimation, Khatri Rao product, minimum redundancy array, nested arrays, spatial smoothing.

\section{INTRODUCTION}

A NTENNA arrays perform spatial sampling of impinging electromagnetic waves which are used to perform improved detection of the source signal or estimate its spatial signature. Direction-of-arrival (DOA) estimation and beamforming are two major applications of the antenna array. However, both of them have been mostly confined to the case of uniform linear arrays (ULA) [1]. The number of sources that can be resolved with a $N$ element ULA using traditional subspace based methods like MUSIC [2] is $N-1$. In this paper, we explore the class of nonuniform arrays and propose

Manuscript received November 19, 2009; accepted April 17, 2010. Date of publication April 29, 2010; date of current version July 14, 2010. The associate editor coordinating the review of this manuscript and approving it for publication was Prof. Maria Sabrina Greco. This work was supported in part by the ONR by Grant N00014-08-1-0709 and by the California Institute of Technology.

The authors are with the California Institute of Technology, Pasadena, CA 91125 USA (e-mail: piyapal@ caltech.edu; ppvnath@systems.caltech.edu.)

Digital Object Identifier 10.1109/TSP.2010.2049264 a novel array structure which is capable of providing a dramatic increase in the degrees of freedom (DOF) and hence can resolve significantly more sources than the actual number of physical sensors. We call this class of arrays as "nested arrays" because they are obtained by combining two or more ULAs with increasing intersensor spacing. We shall demonstrate that using only second-order statistics of the impinging sources, it is possible to obtain $O\left(N^{2}\right)$ DOF from only $O(N)$ physical sensors.

In earlier works, the problem of detecting more sources than sensors has been addressed in different ways. In [3] and [4], it was shown that through the use of minimum redundancy arrays [9] and by constructing an augmented covariance matrix, degrees of freedom can be improved. However, the constructed augmented covariance matrix is not positive semidefinite for finite number of snapshots (and hence violates the condition for being a covariance matrix.) In [5], [6], a transformation of this augmented matrix into a suitable positive definite Toeplitz matrix was suggested and an elaborate algorithm was provided to construct this matrix. However, there are two issues with this approach. To gain more degrees of freedom required for detection of more then $N-1$ sources with $N$ sensors, they rely on the class of minimum redundancy arrays (MRAs), for which, unfortunately, there is no closed form expression for the array geometry and achievable degrees of freedom for a given $N$. The optimum design of such arrays is not easy and in most cases, they are restricted to computer simulations or complicated algorithms for sensor placement [7], [8], [10]-[12]. Also, the algorithm for finding the suitable covariance matrix corresponding to the longer array is a lengthy and complicated iterative algorithm, which converges only to a local optmimum [5], [6]. In [13], the use of fourth-order cumulants was suggested to completely remove the Gaussian noise term and perform better DOA estimation. It was later shown that through the use of fourth-order cumulants, one can also achieve significant increase in degrees of freedom [14]-[16]. But one weakness of this approach is that it is restricted to non-Gaussian sources. Recently, using the concept of Khatri-Rao (KR) product and assuming quasi stationary sources, it has been shown that one can identify up to $2 N-1$ sources using a $N$ element ULA [17] without computing higher-order statistics. It is to be noted that using the augmented array approach of [3] or with the construction of suitable positive definite Toeplitz matrices as done in [5], it will not be possible to obtain this many degrees of freedom using a ULA. But this method, requiring quasi stationary sources, is not applicable to stationary sources. In [18], degrees of freedom were increased by generating a virtual array using a MIMO radar. However, the generation of the virtual array needs active sensing, i.e., both 
transmit and receive antennas, and is not applicable to the case of passive sensing.

In this paper, we develop a new signal model based on the concept of difference co-array in Section II which will help us in performing array processing with increased degrees of freedom in a completely passive scenario. The co-array concept has previously been treated for specific array geometries in [19] and [20]. In the light of the co-array, we shall compare the earlier methods to the more recent one based on K-R product, and bring to light some important differences between them. In Section IV, we propose our nested array structure which can greatly increase the degrees of freedom of the corresponding co-array. We also extend the idea of nesting to multiple levels and a combinatorial optimization problem is next solved to deduce the optimum nesting strategy. As we shall discuss, the extension of the concept of nesting to more than two levels (say $2 K$ levels with $N$ sensors in each level) can easily provide corresponding increase in achievable degrees of freedom to $\left(O\left(N^{2 K}\right)\right)$ when higher than second-order statistics ( $2 K$ th-order statistics) are employed. In comparison to aforementioned existing approaches, our proposed method has several advantages. It will be shown in Section IV that the nested array is extremely easy to construct and it is possible to provide exact closed form expressions for the sensor locations and the degrees of freedom for a given number $(N)$ of sensors, unlike the MRAs used in [3], [5], and [6]. Also, to alleviate the weaknesses of [13] and [17] discussed before, we shall propose a novel spatial smoothing based technique in Section V which utilizes only second-order statistics to exploit the degrees of freedom offered by the array and works well even for stationary sources. Our technique constructs a suitable covariance matrix (which we shall refer to as the spatially smoothed matrix) corresponding to a longer array on which subspace based methods can be applied directly to perform detection estimation of more sources than sensors. Unlike the augmented matrix in [3], the spatially smoothed matrix is guaranteed to be positive semidefinite for any finite number of snapshots and, hence, this spatial smoothing technique constructs a suitable candidate for the covariance matrix of the longer array without resorting to the elaborate iterative techniques adopted in [5] and [6]. Also, unlike MIMO radar, the enhancement in degrees of freedom is achieved entirely through passive sensing. An important advantage of this approach is that, unlike MIMO radar, the increased degrees of freedom can also be used to suppress the jammers. In MIMO radar, since the jammers do not "see" the virtual array, the increased degrees of freedom provided by the virtual array cannot be directly used to null the jammers. As another potential application of the new signal model with nested array, a new approach to beamforming is proposed in Section VI, which directly makes use of the degrees of freedom offered by the co-array. This beamforming spatially filters the powers of the sources (instead of their amplitude) and hence it is inherently nonlinear in nature. Another major advantage of the proposed approach to beamforming is that, assuming perfect knowledge of the signal covariance matrix, it can eliminate noise, which is never possible using conventional linear approach to beamforming. It should be pointed out that the proposed spatial smoothing based method as well as the beamforming are applicable to any array whose difference co-array is a filled ULA and hence they can be applied even to MRAs. In Section VII, extensive simulations are performed to verify all the proposed techniques and compare them against the conventional methods. Finally, Section VIII concludes the paper.

\section{Notations}

Matrices are denoted by capital letters in boldface (e.g., A). Vectors are denoted by lowercase letters in boldface (e.g., a). Superscript ${ }^{H}$ denotes transpose conjugate, whereas superscript * denotes conjugation without transpose. The symbol $\odot$ denotes the Khatri-Rao product [1] between two matrices of appropriate size and the symbol $\otimes$ is used to denote the left Kronecker product [1, p. 1353].

\section{Definitions and Signal Model Based on Difference Co-Array}

Consider a $N$ element possibly nonuniform linear antenna array. Let $\mathbf{a}(\theta)$ be the $N \times 1$ steering vector corresponding to the direction $\theta$ whose $i$ th element is $e^{j(2 \pi / \lambda) d_{i} \sin \theta}$. The sensors are assumed to be placed on a linear grid with $d_{i}$ denoting the position of the $i$ th sensor, which is an integer multiple of the smallest spacing in the underlying grid. The carrier wavelength is denoted by $\lambda$. Let us assume $D$ narrowband sources impinging on this array from directions $\left\{\theta_{i}, i=1,2, \ldots, D\right\}$ with powers $\left\{\sigma_{i}^{2}, i=1,2, \ldots, D\right\}$, respectively. Hence the received signal is

$$
\mathbf{x}[k]=\mathbf{A s}[k]+\mathbf{n}[k]
$$

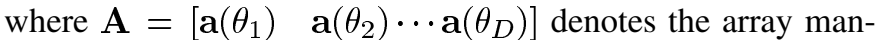
ifold matrix and $\mathbf{s}[k]=\left[s_{1}[k] \quad s_{2}[k] \cdots s_{D}[k]\right]^{T}$ denotes the source signal vector. The noise $\mathbf{n}[k]$ is assumed to be temporally and spatially white, and uncorrelated from the sources. We also assume the sources to be temporally uncorrelated so that the source autocorrelation matrix of $\mathbf{s}[k]$ is diagonal. Then

$$
\begin{aligned}
\mathbf{R}_{\mathbf{x x}} & =E\left[\mathbf{x x}^{H}\right] \\
& =\mathbf{A R}_{\mathbf{s s}} \mathbf{A}^{H}+\sigma_{n}^{2} \mathbf{I} \\
& =\mathbf{A}\left(\begin{array}{cccc}
\sigma_{1}^{2} & & & \\
& \sigma_{2}^{2} & & \\
& & \ddots & \\
& & & \sigma_{D}^{2}
\end{array}\right) \mathbf{A}^{H}+\sigma_{n}^{2} \mathbf{I} .
\end{aligned}
$$

Now, following [17], we vectorize $\mathbf{R}_{\mathbf{x x}}$ to get the following vector:

$$
\begin{aligned}
\mathbf{z} & =\operatorname{vec}\left(\mathbf{R}_{\mathbf{x x}}\right)=\operatorname{vec}\left[\sum_{i=1}^{D} \sigma_{i}^{2}\left(\mathbf{a}\left(\theta_{i}\right) \mathbf{a}^{H}\left(\theta_{i}\right)\right)\right]+\sigma_{n}^{2} \overrightarrow{\mathbf{1}}_{n} \\
& =\left(\mathbf{A}^{*} \odot \mathbf{A}\right) \mathbf{p}+\sigma_{n}^{2} \overrightarrow{\mathbf{1}}_{n}
\end{aligned}
$$

where $\mathbf{p}=\left[\begin{array}{lll}\sigma_{1}^{2} & \sigma_{2}^{2} \cdots \sigma_{D}^{2}\end{array}\right]^{T}$ and $\overrightarrow{\mathbf{1}}_{n}=\left[\begin{array}{ll}\mathbf{e}_{\mathbf{1}}^{T} & \mathbf{e}_{\mathbf{2}}{ }^{T} \cdots \mathbf{e}_{\mathbf{N}}^{T}\end{array}\right]^{T}$ with $\mathbf{e}_{i}$ being a column vector of all zeros except a 1 at the $i$ th position. Comparing it with (1), we can say that $\mathbf{z}$ in (3) behaves like the received signal at an array whose manifold is given by $\mathbf{A}^{*} \odot \mathbf{A}$ where $\odot$ denotes the KR product. The equivalent source signal vector is represented by $\mathbf{p}$ and the noise becomes a deterministic vector given by $\sigma_{n}^{2} \overrightarrow{\mathbf{1}}_{n}$. The distinct 
rows of $\mathbf{A}^{*} \odot \mathbf{A}$ behave like the manifold of a (longer) array whose sensor locations are given by the distinct values in the set $\left\{\overrightarrow{\mathbf{x}_{i}}-\overrightarrow{\mathbf{x}_{j}}, 1 \leq i, j \leq N\right\}$ where $\overrightarrow{\mathbf{x}_{i}}$ denotes the position vector of the $i$ th sensor of the original array. This array is precisely the difference co-array of the original array [19]. Hence instead of (1), we can apply DOA estimation to the data in (3) and work with the difference co-array instead of the original array. The difference co-array thus occurs naturally in problems involving second-order statistics of the received signal. Even the methods based on fourth-order cumulants end up exploiting the degrees of freedom of the difference co-array as was pointed out in [14]-[16].

\section{The Co-ARRAY PERSPECTIVE}

In this section, we shall first briefly discuss the concept of co-array along with its important properties and then compare the achievable degrees of the different source localization methods discussed earlier, by explaining to what extent those algorithms can exploit the degrees of freedom provided by the co-arrays.

Definition 1: (Difference Co-Array): Let us consider an array of $N$ sensors, with $\overrightarrow{\mathbf{x}_{i}}$ denoting the position vector of the $i$ th sensor. Define the set

$$
D=\left\{\overrightarrow{\mathbf{x}_{i}}-\overrightarrow{\mathbf{x}}_{j}\right\}, \quad \forall i, j=1,2, \ldots, N .
$$

In our definition of the set $D$, we allow repetition of its elements. We also define the set $D_{u}$ which consists of the distinct elements of the set $D$. Then, the difference co-array of the given array is defined as the array which has sensors located at positions given by the set $D_{u}$.

The number of elements in the difference co-array (given by the set $D_{u}$ ) directly decides the distinct values of the cross correlation terms in the covariance matrix of the signal received by an antenna array. Using these distinct cross correlation terms judiciously in different ways, one can substantially increase the number of sources that can be detected by the array. Each such technique actually amounts to using a part or whole of the resulting difference co-array, instead of the original array, to perform the DOA estimation. Of course, the maximum degrees of freedom that can be attained is limited by the number of elements present in the co-array. There is a related concept of "sum co-array" which we do not consider here as it does not arise naturally in computation of the covariance matrix of the received signal.

Definition 2: (Weight Function): Define an integer valued function $w: D_{u} \longrightarrow \mathbb{N}^{+}$such that $w(\overrightarrow{\mathbf{d}})=$ no. of occurences of $\overrightarrow{\mathbf{d}}$ in $D, \overrightarrow{\mathbf{d}} \in D_{u}$, where $\mathbb{N}^{+}$is the set of positive integers. The weight function $w(\overrightarrow{\mathbf{d}})$ denotes the number of times $\overrightarrow{\mathbf{d}}$ occurs.

\section{A. Properties of Difference Co-Array}

Let us consider any $N$ element array (possibly nonlinear). Then the following are true for the weight function $w($.$) corre-$ sponding to its difference co-array:

1) $w(\overrightarrow{\mathbf{0}})=N$

2) $1 \leq w(\overrightarrow{\mathbf{d}}) \leq N-1 \quad \forall \overrightarrow{\mathbf{d}} \in D_{u} \backslash\{\overrightarrow{\mathbf{0}}\}$

3) $w(\overrightarrow{\mathbf{d}})=w(-\overrightarrow{\mathbf{d}}), \quad \forall \overrightarrow{\mathbf{d}} \in D_{u}$

4) $\sum_{\overrightarrow{\mathbf{d}} \in D_{u}, \overrightarrow{\mathbf{d}} \neq \overrightarrow{0}} w(\overrightarrow{\mathbf{d}})=N(N-1)$
Property (1) is due to the fact that $\overrightarrow{\mathbf{d}}=\overrightarrow{\mathbf{0}}$ occurs whenever $\overrightarrow{\mathbf{x}_{i}}=\overrightarrow{\mathbf{x}_{j}}$ in (4) and that happens exactly $N$ times for $i=j=$ $1,2, \ldots, N$.

Property (2) can be verified as follows: Say the value $\overrightarrow{\mathbf{d}} \neq \overrightarrow{\mathbf{0}}$ occurs as the difference between the $i$ th and the $j$ th sensors, i.e., $\overrightarrow{\mathbf{d}}=\overrightarrow{\mathbf{x}_{i}}-\overrightarrow{\mathbf{x}_{j}}$. Then, $\overrightarrow{\mathbf{d}} \neq \overrightarrow{\mathbf{x}_{i}}-\overrightarrow{\mathbf{x}_{k}}$ for any $k \neq j$. Therefore $\overrightarrow{\mathbf{d}}$ can occur only once as we consider the set of differences in position between a given sensor (say, $i$ th) and the remaining sensors. So the maximum number of times it can occur is once, for each value of $i=1,2, \ldots, N-1$, i.e., it can occur a maximum of $N-1$ times. For example, in a $N$ element ULA with unit spacing, the difference of 1 occurs exactly $N-1$ times.

Property (3) is easily verifiable since whenever $\overrightarrow{\mathbf{d}}=\overrightarrow{\mathbf{x}_{i}}-$ $\overrightarrow{\mathbf{x}_{j}}$ occurs, correspondingly we also get the position difference $-\overrightarrow{\mathbf{d}}=\overrightarrow{\mathbf{x}_{j}}-\overrightarrow{\mathbf{x}_{i}}$. Hence the values $\overrightarrow{\mathbf{d}}$ and $-\overrightarrow{\mathbf{d}}$ occur with equal frequency.

Property (4) can be verified as follows: The LHS indicates the sum of occurences of all possible nonzero position differences $\overrightarrow{\mathbf{d}} \in D_{u}$. The total number of times all position differences can occur is exactly equal to all possible permutations, taken two at a time from the set $\left\{\overrightarrow{\mathbf{x}}_{i}, i=1,2 \ldots, N\right\}$, which is equal to $N(N-1)$.

It is to be noted that the cardinality of $D_{u}$ for a given array gives the degrees of freedom that can be obtained from the difference co-array associated with that array. However, from property (4) above, we can immediately conclude that the maximum degrees of freedom that can be obtainable from a difference co-array for a $N$ element array with any geometry, is

$$
\mathrm{DOF}_{\max }=N(N-1)+1 .
$$

Thus we see that if we use second-order statistics, then, by exploiting the degrees of freedom (DOF) of the difference co-array, there is a possibility that we can get $O\left(N^{2}\right)$ degrees of freedom using only $O(N)$ physical elements. $\mathrm{DOF}_{\max }$ gives a global upper bound on the maximum DOF achievable from the difference co-array over all classes of arrays. Also there is a trade-off between the degrees of freedom and the value of the weight function which is clear from Property (4) above. If a difference occurs more than once (i.e., $w(\overrightarrow{\mathbf{d}})>1$ for some $\overrightarrow{\mathbf{d}}$ ), then it implies a drop in the overall cardinality (and thereby the degrees of freedom) of $D_{u}$.

\section{B. Computing the Weight Function}

Given a linear array with $d$ as the minimum spacing of the underlying grid on which sensors are assumed to be located, define the function $c(m)$ which takes a value 1 if there is a sensor located at $m d$ and 0 otherwise. Then the weight function $w(n)$ can be computed as the convolution:

$$
w(n)=\left(c \circledast c^{-}\right)(n) .
$$

where $c^{-}(n)=c(-n)$. As an example, the difference co-array of an $N$ element ULA is another ULA with $2 N-1$ elements. The difference co-array of a uniform circular array (UCA) is a set of concentric UCAs with $N(N-1)+1$ degrees of freedom when $N$ is odd. Minimum redundancy arrays form a class of nonuniform arrays which have the longest difference co-arrays 
under the constraint that the difference co-array is a ULA. However, the sensor locations and degrees of freedom of such arrays cannot be computed in closed form for any arbitrary $N$ and they are found through computer search [7], [8].

\section{Comparison of Underdetermined DOA Estimation Techniques From the Co-Array Perspective}

1) Augmented Matrix Approach [3], [4], [11]:

This method exploits the difference co-array of a minimum redundancy array but effectively uses only one half of the difference co-array, because the negative differences are used up to make the augmented matrix Hermitian. Hence, it can attain $M / 2$ degrees of freedom where $M$ is the cardinality of $D_{u}$. Also, in case of ULA, this method cannot exploit the additional degrees of freedom provided by the difference co-array of the ULA because it only uses one half of the co-array. So it cannot identify sources beyond the traditional limit of $N-1$ for a $N$ element ULA. The modification to [3] that was suggested in [5], [6] also has the same limitation. They assume that the maximum number of resolvable sources that can be obtained from any $N$ element array is $N(N-1) / 2+1$ (which is because they aim at constructing a Hermitian Toeplitz matrix) whereas the actual difference co-array provides twice as many degrees of freedom. So using some other suitable algorithm, there is a possibility that this additional freedom might be exploited and even with a $N$ element ULA, one can resolve $2 N-2$ sources (see below).

2) Fourth-order cumulants [13]-[16]: Here the effective steering vector becomes $\mathbf{a}_{e f f}=\mathbf{a} \otimes \mathbf{a}^{*}$, as given in [13] and [16]. Thus $\mathbf{a}_{\text {eff }}$ behaves exactly like the steering vector corresponding to the entire difference co-array of the original array, where each row represents an element of the co-array. Depending on the weight function of the co-array, the row corresponding to the $n$th element of the co-array occurs $w(n)$ times in $\mathbf{a}_{e f f}$. Nevertheless, it captures all degrees of freedom offered by the entire co-array and hence even for an ULA with $N$ sensors, it can resolve up to $2 N-2$ sources. However, a drawback of this method is that it is applicable only to cases where the source signals are non-Gaussian. Also, one needs to compute the fourth-order cumulants of the received signal which might require large number of snapshots and longer computation time.

3) KR product based MUSIC: [17] This recent method exploits quasi-stationarity of source signals to construct a full rank matrix whose rank is given by the rank of the effective array manifold $\mathbf{A}_{\text {eff }}=\mathbf{A}^{*} \odot \mathbf{A}$ where $\odot$ denotes the KR product. $\mathbf{A}_{\text {eff }}$ behaves like the manifold of the entire difference co-array and hence it is inherently capable of exploiting the full degrees of freedom offered by the difference co-array. The authors considered a $N$ element ULA and provided a Krushkal rank based argument to prove that it can identify upto $2 N-2$ sources. However such argument is not necessary because the identifiability condition becomes obvious when we view it in the light of the co-array of a ULA. The advantage of this method is that it uses only second-order statistics of the data and hence has no problem in handling Gaussian sources. However, it requires quasi-stationarity to ensure that the constructed matrix is full rank.

\section{The ConcePt of NeSted ARRAY: Degrees of FreEDOM AND OPTIMIZATION}

As we observed in Section III, a key idea behind the ability to resolve more sources than physical sensors, is to use a possibly nonuniform array so that its difference co-array has significantly more degrees of freedom than the original array. In [3] and [5], classes of Minimum Redundancy arrays (MRAs) were used to achieve this purpose. However, the main problem is that there is no simple way to construct these arrays and it requires extensive computer search [1], [10], [12]. Hence, there is no way to predict the attainable degrees of freedom of MRAs for a given $N$ other than the fact that it is always strictly less than $N(N-1)+1$ [1]. So, much of the work on underdetermined DOA estimation problems have been performed considering specific examples of MRAs instead of a general class.

In this section, we attempt to provide a solution to this problem by proposing a class of "nested arrays." This array structure, as we shall show, can be generated very easily in a systematic fashion and we can exactly predict degrees of freedom of its co-array for a given $N$. Moreover, with this class, it is indeed possible to generate $O\left(N^{2}\right)$ degrees of freedom from $O(N)$ physical elements. It is also easy to extend this idea of nesting to higher dimensions. A similar increase in degrees of freedom has been achieved in MIMO radar using the virtual array concept through active sensing. But here we will show that even in the passive scenario, it is possible to exploit such nested structures and attain even more degrees of freedom compared to the corresponding MIMO radar with same number of physical elements. The "two level" nested array as we shall define below, is similar to the array structure originally proposed by Bracewell (see [28]). The combination of the transmitting and the receiving arrays in MIMO radar also has a similar structure. However, we shall generalize the structure to beyond 2 levels to systematically increase the degrees of freedom.

\section{A. MIMO Radar and the Virtual Array}

Consider a collocated MIMO radar with an $M$ element ULA as the transmitter and an $N$ element ULA as the receiver. The transmitter transmits $M$ orthogonal waveforms, each through one antenna which are separately extracted through matched filtering at the receiver. If the spacing at the transmitter is made $M$ times that at the receiver, then we can make the signals at the output of the matched filter at the receiver behave as if they have been received by a virtual array of NM sensors [10], [18], [21]. This virtual array is essentially the convolution of the two ULAs and it provides a convenient way to attain $N M$ degrees of freedom using only $N+M$ physical sensors. The sensor positions in the new virtual array are given by the set $V A=\left\{\mathbf{x}_{T i}+\mathbf{x}_{R} j, \quad i=1, \ldots, M, \quad j=1, \ldots, N\right\}$ where $\mathbf{x}_{T i}$ and $\mathbf{x}_{R} j$ denote the position of the $i$ th transmit and the $j$ th receive antenna, respectively. Comparing with (4), we have the sum of positions instead of the differences. This is somewhat similar to the sum co-array discussed in [19], the difference 


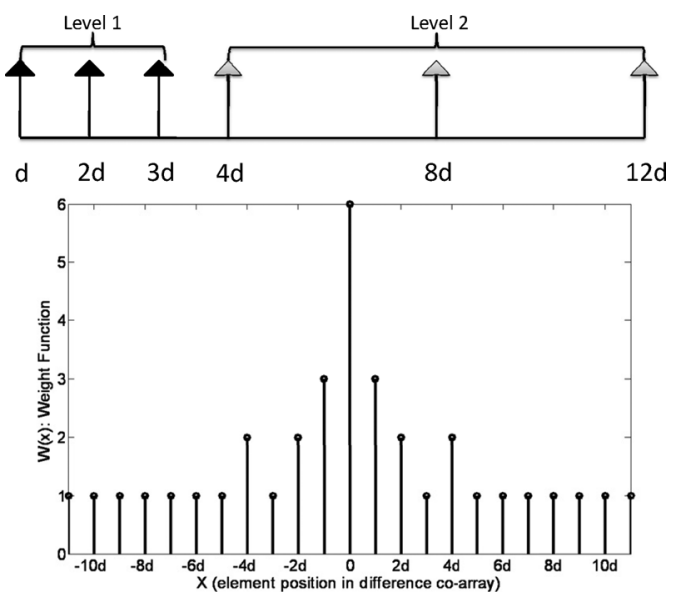

Fig. 1. A 2 level nested array with 3 sensors in each level (top), and the weight function of its difference co-array (bottom).

being, the sum is between two different sets instead of between the same set. For an arbitrary array, the geometry of the sum and the difference co-arrays in general will be quite different and so will be their degrees of freedom.

\section{B. Two Level Nested Passive Array}

A two-level nested array is basically a concatenation of two ULAs: inner and outer where the inner ULA has $N_{1}$ elements with spacing $d_{1}$ and the outer ULA has $N_{2}$ elements with spacing $d_{2}$ such that $d_{2}=\left(N_{1}+1\right) d_{1}$. More precisely it is a linear array with sensors locations given by the union of the sets $S_{\text {inner }}=\left\{m d_{1}, \quad m=1,2, \ldots, N_{1}\right\}$ and $S_{\text {outer }}=\left\{n\left(N_{1}+1\right) d_{1}, \quad n=1,2, \ldots, N_{2}\right\}$. Fig. 1 illustrates the nested array. It is similar to the union of the transmit and the receive arrays of a MIMO radar and uses the same number of sensors. However here we use a single such array for receiving only (passive sensing) instead of active sensing as done in MIMO radar.

Fig. 1 also shows the difference co-array of this nested array. Two important observations about the co-array are:

- It is a filled ULA with $2 N_{2}\left(N_{1}+1\right)-1$ elements whose positions are given by the set $S_{c a}$ defined as

$$
S_{c a}=\left\{n d_{1}, n=-M, \ldots, M, \quad M=N_{2}\left(N_{1}+1\right)-1\right\}
$$

- Comparing with the virtual array of the MIMO radar, we find that it has twice as many degrees of freedom though both use the same number of physical sensors. Also, the proposed nested array can be used as a passive array, i.e., the entire array is used for "receive only", and yet we can successfully exploit the degrees of freedom of its co-array (as we shall demonstrate when we perform DOA estimation and beamforming with this array) as opposed to the MIMO radar situation where the longer virtual array can only be realized through "active sensing".

Thus we demonstrated that with a two-level nested array, we can attain $2 N_{2}\left(N_{1}+1\right)-1$ freedoms in the co-array using only $N_{1}+N_{2}$ elements. This indeed gives us a systematic way to increase the degrees of freedom of the co-array, as opposed to the MRAs which need computer search to find those array

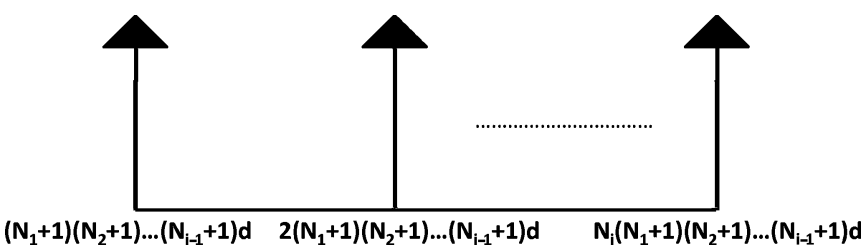

Fig. 2. The $i$ th nesting level containing $N_{i}$ sensors in a $K$-level nested linear array.

structures. We can further optimize the distribution of sensors in the two levels by finding $N_{1}, N_{2}$ that maximize the total degrees of freedom, $2 N_{2}\left(N_{1}+1\right)-1$, under the constraint of fixed total number of sensors, i.e., $N_{1}+N_{2}=N$. The solution (using AM-GM inequalities) can be verified as:

\begin{tabular}{|c|c|c|}
\hline $\mathrm{N}$ & optimal $N_{1}, N_{2}$ & DOF \\
\hline even & $N_{1}=N_{2}=\frac{1}{2} N$ & $\frac{N^{2}-2}{2}+N$ \\
\hline odd & $N_{1}=\frac{N-1}{2}, \quad N_{2}=\frac{N+1}{2}$ & $\frac{N^{2}-1}{2}+N$ \\
\hline
\end{tabular}

Hence, using 2 level of nesting, we can obtain little over half of the maximum limit in (5). So now we examine how far we can increase the degrees of freedom of the co-array by extending the nesting strategy to more than two levels.

\section{C. $K$ Levels of Nesting}

A ' $K$-level' nested linear array, parameterized by $K, N_{1}, N_{2}, \ldots, N_{K} \in \mathbb{N}^{+}$, is defined as one where the sensor positions are given by the set $\mathrm{S}_{(K \text {-level })}=\bigcup_{i=1}^{K} \mathrm{~S}_{i}$ where

$$
\begin{aligned}
\mathrm{S}_{i} & =\left\{n d \prod_{j=1}^{i-1}\left(N_{j}+1\right), \quad n=1,2, \ldots, N_{i}\right\}, \quad i=2, \ldots, K \\
\mathrm{~S}_{1} & =\left\{n d, \quad n=1, \ldots, N_{1}\right\} .
\end{aligned}
$$

The structure of the $i$ th nesting level $(i \neq 1)$ in a $K$-level nested array is depicted in Fig. 2. So, the " $K$ "-level nested array is essentially a union of $K$ ULAs with $N_{i}$ sensors in the $i$ th level of nesting and the intersensor spacing in the $i$ th level is $\left(N_{i-1}+1\right)$ times the inter-sensor spacing in the $(i-1)$ th level. Note that the total number of physical sensors in the nested array is $\sum_{i=1}^{K} N_{i}=N$.

It can be verified using the convolution formula in (6) that the degrees of freedom in the corresponding difference co-array is

$$
\begin{aligned}
\operatorname{DOF}_{K}= & 2\left\{\left[N_{2}\left(N_{1}+1\right)-1\right]+\left[\left(N_{3}-1\right)\left(N_{1}+N_{2}+1\right)\right.\right. \\
& \left.+\left(N_{1}+1\right)\right]+\cdots+\left[( N _ { K } - 1 ) \left(N_{1}+N_{2}+N_{3}+\right.\right. \\
& \left.\left.\left.\cdots+N_{K-1}+1\right)+\left(N_{1}+N_{2}+\cdots+N_{K-2}+1\right)\right]\right\}+1
\end{aligned}
$$

which can be simplified to

$$
\mathrm{DOF}_{K}=2\left(\sum_{i=1}^{K} \sum_{j=i+1}^{K} N_{i} N_{j}+N_{K}-1\right)+1 .
$$

The corresponding difference co-array, however, is not a filled ULA for more than two stages of nesting. 


\section{Optimization of the $N$ Level Nested Array}

Given a number of sensors, $N$, we would like to know the number of nested levels and also the number of sensors per nesting level, which will maximize the degrees of freedom of the nested array. This can be cast as the following optimization problem:

$$
\begin{aligned}
& \max _{K \in \mathbb{N}^{+}} \max _{N_{1}, \ldots, N_{K} \in \mathbb{N}^{+}} \operatorname{DOF}_{K} \\
& \text { subject to: } \sum_{i=1}^{K} N_{i}=N .
\end{aligned}
$$

Since the variables $K, N_{1}, \ldots, N_{K}$ are all positive integers, this is a combinatorial optimization problem. However, we will provide a closed form solution to this problem using a simple observation and thereby deduce the structure of the optimally nested array. The solution to this problem is given by the following theorem

Theorem 1: Given a number $N$ of sensors, the optimal number of nesting levels $K$ and the number of sensors per nesting level are given by

$$
\begin{aligned}
K & =N-1 \\
\text { and } \quad N_{i} & =\left\{\begin{array}{ll}
1, & i=1,2,3, \ldots, K-1 \\
2, & i=K
\end{array} .\right.
\end{aligned}
$$

The corresponding difference array is a nonuniform linear array with degrees of freedom given by $\mathrm{DOF}_{\text {opt }}=N(N-1)+1$ which is same as the upper bound in (5).

Proof: See Appendix .

Corollary 1: Structure of the Optimally Nested Array: Substituting these optimum values into (8), it can be verified that the optimum nested array has sensors located at the positions given by the set $\mathrm{S}_{\mathrm{opt}}=\left\{d, 2 d, 4 d, 8 d, \ldots, 2^{N-1} d\right\}$.

Hence we have this interesting observation: The optimally nested array is one with exponential spacing.

\section{Remarks}

1) Compared to the minimum redundancy arrays, our two level nested array will always produce less degrees of freedom since the minimum redundancy arrays are optimized to produce the longest difference co-array under the constraint that the difference co-array is a ULA. However, the structure of a MRA for a given $N$ cannot be exactly predicted and it can only be found through numerical simulations individually for each $N$. In contrast, the nested arrays are easy to construct and the sensor positions and degrees of freedom are analytically tractable and are also $O\left(N^{2}\right)$.

2) Extending the nesting strategy beyond two levels fails to produce a difference co-array which is a ULA (i.e., holes appear). Same is true for the optimally nested array which is exponentially spaced. This is because the difference array is associated with the second-order statistics of the received data. So, extending beyond two levels of nesting will have a nice advantage if we consider higher-order statistics. For example, if we consider fourth-order statistics, then instead of the difference co-array, we shall be interested in the difference of the difference set. In such a situation, a 4 level nested array with $N$ elements in each level, will beautifully produce a filled ULA with $O\left(N^{4}\right)$ elements. Thus, an important advantage of this nesting strategy is that it easily extends to the case of higher-order statistics, producing consistent increase in degrees of freedom. In future, we shall explore this advantage in greater detail. It is to be noted that MRAs cannot yield $O\left(N^{4}\right)$ degrees of freedom with fourth-order statistics because the difference array is a ULA.

3) The optimally nested array belongs to a special class of array, namely, the nonredundant array. Non redundant arrays are the ones for which every difference in the difference co-array appears only once (except the zero lag which has to appear $N$ times). Hence, by Property 4 in Section III, it achieves the maximum value of degrees of freedom given by (5) for any value of $N$. It is interesting to note that while the two level nested array belongs to the class of "no-hole" arrays (arrays with no holes but with redundancies), the optimally nested array is a "nonredundant array" (arrays which have no redundancies but have holes).

For comparison, we enlist the degrees of freedom in the difference co-array of the MRAs [1], 2 level nested array and the upper bound in (5) (achieved by the optimally nested array) for different values of $N$ :

\begin{tabular}{|c|c|c|c|}
\hline $\mathrm{N}$ & MRA & 2 level & Upper Bound \\
\hline 4 & 13 & 11 & 13 \\
\hline 8 & 47 & 39 & 57 \\
\hline 9 & 59 & 49 & 73 \\
\hline 10 & 73 & 59 & 91 \\
\hline 15 & 159 & 127 & 211 \\
\hline
\end{tabular}

\section{Applications of the NeSted ARRAY IN DOA ESTIMATION OF MORE SOURCES THAN SENSORS}

As discussed in Section III, the increased degrees of freedom offered by the co-array were exploited by different techniques such as augmented matrix approach [3], [5], fourth-order cumulant based methods [13] and the recent quasi stationary signal based method [17]. However, as we pointed out earlier, all of them have limitations. Hence, in the following section, we propose an alternative way to exploit the degrees of freedom by applying the spatial smoothing technique [22] in a novel fashion. This approach guarantees to produce a positive semidefinite covariance matrix corresponding to the co-array even for finite snapshots (thereby overcoming the limitation of [3] without resorting to the multiple iterations suggested in [5]). Also, unlike the methods based on fourth-order cumulants, our proposed approach would work even in the case of Gaussian signals and unlike [17], it is equally applicable for stationary signals.

\section{A. Spatial Smoothing Based DOA Estimation}

We propose a spatial smoothing based approach for exploiting the degrees of freedom of the difference co-array. It is to be noted that we do not use spatial smoothing for decorrelating uncorrelated sources, as it is done in the conventional 
sense [22], [23]. Rather, it is used as a technique to build up the rank of an observation matrix so that we do not need to either use the fourth-order cumulant or assume quasi stationarity of signals. Note that spatial smoothing works only for a ULA and so we shall focus on the two-level nested array for applying this technique since its difference co-array is a filled ULA. It should be remembered that application of the proposed spatial smoothing based method is not restricted to nested arrays and it can be applied to any array whose difference co-array is a filled ULA (including the MRA).

Consider the signal model in Section II given by (3) for a two level nested array with $N / 2$ sensors in each level. We assume $N$ to be even, similar arguments can be applied for the odd case as well. The dimension of the new array manifold $\mathbf{A}^{*} \odot \mathbf{A}$ is $N^{2} \times D$ but it has precisely as many distinct rows as the number of degrees of freedom of the difference co-array which is $\left(N^{2}-2\right) / 2+N$ for 2 level nested array for even $N$. Since the difference co-array in this case is a filled ULA, $\mathbf{A}^{*} \odot \mathbf{A}$ is like a Vandermonde matrix with $\left(N^{2}-2\right) / 2+N$ distinct rows and hence its rank is $D$ as long as $D \leq\left(N^{2}-2\right) / 2+N$. However the equivalent source signal vector $\mathbf{p}$ for this co-array consists of the powers $\sigma_{i}^{2}$ of the actual sources and hence they behave like fully coherent sources. In [17], this problem was taken care of by assuming the source powers to vary in different quasi stationary intervals and these were collected to build a full rank matrix. Here, we propose an alternate solution by using the well known technique of spatial smoothing that is applied for coherent sources, to the new signal model given by (3). We should like to emphasize that the spatial smoothing technique which is usually applied for ULAs, might also be used for the difference co-array (which is not a ULA) of the optimally nested array after doing some preprocessing like interpolation as suggested by [24].

To apply spatial smoothing, first let us construct a new matrix $\mathbf{A}_{1}$ of size $\left(\left(N^{2}-2\right) / 2+N\right) \times D$ from $\mathbf{A}^{*} \odot \mathbf{A}$ where we have removed the repeated rows (after their first occurrence) and also sorted them so that the $i$ th row corresponds to the sensor location $\left(-N^{2} / 4-N / 2+i\right) d$ in the difference co-array of the 2 level nested array. This is equivalent to removing the corresponding rows from the observation vector $\mathbf{z}$ and sorting them to get a new vector $\mathbf{z}_{1}$ given by

$$
\mathbf{z}_{\mathbf{1}}=\mathbf{A}_{\mathbf{1}} \mathbf{p}+\sigma_{n}^{2} \overrightarrow{\mathbf{e}}^{\prime}
$$

where $\overrightarrow{\mathbf{e}}^{\prime} \in \mathbb{R}^{\left(\left(N^{2}-2\right) / 2+N\right) \times 1}$ is a vector of all zeros except a 1 at the $\left(N^{2} / 4+N / 2\right)$ th position. It can be verified that due to the above mentioned sorting and replacement of repeated rows, the deterministic noise vector changes from $\overrightarrow{\mathbf{1}}_{n}$ in (3) to $\overrightarrow{\mathbf{e}}^{\prime}$.

From (7), the difference co-array of this 2 level nested array has sensors located from $\left(-N^{2} / 4-N / 2+1\right) d$ to $\left(N^{2} / 4+\right.$
$N / 2-1) d$. We now divide this co-array into $N^{2} / 4+N / 2$ overlapping subarrays, each with $N^{2} / 4+N / 2$ elements, where the $i$ th subarray has sensors located at

$$
\left\{(-i+1+n) d, \quad n=0,1, \ldots, \frac{N^{2}}{4}+\frac{N}{2}-1\right\} .
$$

The $i$ th subarray corresponds to the $\left(N^{2} / 4+N / 2-i+1\right)$ th to $\left(\left(N^{2}-2\right) / 2+N-i+1\right)$ th rows of $\mathbf{z}_{\mathbf{1}}$ which we denote as

$$
\mathbf{z}_{\mathbf{1 i}}=\mathbf{A}_{\mathbf{1 i}} \mathbf{p}+\sigma_{n}^{2} \dot{e}_{\mathbf{i}}
$$

where $\mathbf{A}_{1 \mathbf{i}}$ is a $\left(N^{2} / 4+N / 2\right) \times D$ matrix consisting of the $\left(N^{2} / 4+N / 2-i+1\right)$ th to $\left(\left(N^{2}-2\right) / 2+N-i+1\right)$ th rows of $\mathbf{A}_{\mathbf{1}}$ and $\mathbf{e}_{\mathbf{i}}$ is a vector of all zeros except a 1 at the $i$ th position. It is easy to check that

$$
\mathbf{z}_{1 \mathbf{i}}=\mathbf{A}_{\mathbf{1 1}} \boldsymbol{\Phi}^{i-1} \mathbf{p}+\sigma_{n}^{2} \dot{\mathbf{e}}_{\mathbf{i}}
$$

where (see the equation at the bottom of the page). Define

$$
\begin{aligned}
\mathbf{R}_{\mathbf{i}} \triangleq \mathbf{z}_{\mathbf{1 i}} \mathbf{z}_{\mathbf{1 i}}{ }^{H} & =\mathbf{A}_{\mathbf{1 1}} \boldsymbol{\Phi}^{i-1} \mathbf{p} \mathbf{p}^{H}\left(\boldsymbol{\Phi}^{i-1}\right)^{H} \mathbf{A}_{\mathbf{1 1}}{ }^{H}+\sigma_{n}^{4} \mathbf{e}_{\mathbf{i}} \dot{\mathbf{e}}_{\mathbf{i}}^{H} \\
& +\sigma_{n}^{2} \mathbf{A}_{\mathbf{1 1}} \boldsymbol{\Phi}^{i-1} \mathbf{p e ́}_{\mathbf{i}}^{H}+\sigma_{n}^{2} \dot{\mathbf{e}}_{\mathbf{i}} \mathbf{p}^{H}\left(\boldsymbol{\Phi}^{i-1}\right)^{H} \mathbf{A}_{\mathbf{1 1}}{ }^{H} .
\end{aligned}
$$

Taking the average of $\mathbf{R}_{\mathbf{i}}$ over all $i$, we get

$$
\mathbf{R}_{\mathrm{ss}} \triangleq \frac{1}{\left(\frac{N^{2}}{4}+\frac{N}{2}\right)} \sum_{\mathbf{i}=1}^{N^{2} / 4+N / 2} \mathbf{R}_{\mathbf{i}} .
$$

We call the matrix $\mathbf{R}_{\mathbf{s s}}$ as the spatially smmoothed matrix and it enables us to perform DOA estimation of $O\left(N^{2}\right)$ sources with $N$ sensors, as given by the following theorem:

Theorem 2: The matrix $\mathbf{R}_{\mathrm{ss}}$ as defined in (12) can be expressed as $\mathbf{R}_{\mathbf{s s}}=\hat{\mathbf{R}}^{2}$ where

$$
\hat{\mathbf{R}}=\frac{1}{\sqrt{\frac{N^{2}}{4}+\frac{N}{2}}}\left(\mathbf{A}_{\mathbf{1 1}} \boldsymbol{\Lambda} \mathbf{A}_{\mathbf{1 1}}{ }^{H}+\sigma_{n}^{2} \mathbf{I}\right)
$$

has the same form as the covariance matrix of the signal received by a longer ULA consisting of $N^{2} / 4+N / 2$ sensors and hence by applying MUSIC on $\mathbf{R}_{\mathrm{ss}}$, upto $N^{2} / 4+N / 2-1$ sources can be identified.

Proof: From (12), we get

$$
\begin{aligned}
\mathbf{R}_{\mathbf{S S}}= & \frac{1}{\left(\frac{N^{2}}{4}+\frac{N}{2}\right)} \sum_{\mathbf{i}=1}^{N^{2} / 4+N / 2} \mathbf{R}_{\mathbf{i}} \\
= & \frac{1}{\left(\frac{N^{2}}{4}+\frac{N}{2}\right)}\left[\mathbf{A}_{\mathbf{1 1}} \mathbf{C C}^{H} \mathbf{A}_{\mathbf{1 1}}{ }^{H}+\sigma_{n}^{4} \mathbf{I}\right. \\
& \left.+\sigma_{n}^{2} \mathbf{A}_{\mathbf{1 1}} \mathbf{C}+\sigma_{n}^{2} \mathbf{C}^{H} \mathbf{A}_{\mathbf{1 1}}{ }^{H}\right]
\end{aligned}
$$

$$
\boldsymbol{\Phi}=\left(\begin{array}{llll}
e^{-j(2 \pi / \lambda) d \sin \theta_{1}} & & & \\
& e^{-j(2 \pi / \lambda) d \sin \theta_{2}} & & \\
& & \ddots & \\
& & & e^{-j(2 \pi / \lambda) d \sin \theta_{D}}
\end{array}\right)
$$


where

$$
\mathbf{C}=\left(\begin{array}{cccc}
\sigma_{1}^{2} & & & \\
& \sigma_{2}^{2} & & \\
& & \ddots & \\
& & & \sigma_{D}^{2}
\end{array}\right)\left(\begin{array}{cccc}
1 & \nu_{1} & \cdots & \nu_{1}^{\left(N^{2} / 4+N / 2-1\right)} \\
1 & \nu_{2} & \cdots & \nu_{2}^{\left(N^{2} / 4+N / 2-1\right)} \\
\vdots & \vdots & \vdots & \vdots \\
1 & \nu_{D} & \cdots & \nu_{D}^{\left(N^{2} / 4+N / 2-1\right)}
\end{array}\right)
$$

where

$$
\nu_{n}=e^{-j \frac{2 \pi}{\lambda} n d \sin \left(\theta_{i}\right)} .
$$

Noticing that

$$
\mathbf{A}_{\mathbf{1 1}}{ }^{H}=\left(\begin{array}{cccc}
1 & \nu_{1} & \cdots & \nu_{1}^{\left(N^{2} / 4+N / 2-1\right)} \\
1 & \nu_{2} & \cdots & \nu_{2}^{\left(N^{2} / 4+N / 2-1\right)} \\
\vdots & \vdots & \vdots & \vdots \\
1 & \nu_{D} & \cdots & \nu_{D}^{\left(N^{2} / 4+N / 2-1\right)}
\end{array}\right)
$$

we can rewrite (13) as

$$
\begin{aligned}
\mathbf{R}_{\mathbf{s s}}= & \frac{1}{\frac{N^{2}}{4}+\frac{N}{2}}\left[\mathbf{A}_{\mathbf{1 1}} \Lambda \mathbf{A}_{\mathbf{1 1}}{ }^{H} \mathbf{A}_{\mathbf{1 1}} \Lambda \mathbf{A}_{\mathbf{1 1}}{ }^{H}\right. \\
& \left.+\sigma_{n}^{4} \mathbf{I}+2 \sigma_{n}^{2} \mathbf{A}_{\mathbf{1 1}} \Lambda \mathbf{A}_{\mathbf{1 1}}{ }^{H}\right] \\
= & \frac{1}{\left(\frac{N^{2}}{4}+\frac{N}{2}\right)}\left(\mathbf{A}_{\mathbf{1 1}} \boldsymbol{\Lambda} \mathbf{A}_{\mathbf{1 1}}{ }^{H}+\sigma_{n}^{2} \mathbf{I}\right)^{2}
\end{aligned}
$$

where

$$
\Lambda=\left(\begin{array}{cccc}
\sigma_{1}^{2} & & & \\
& \sigma_{2}^{2} & & \\
& & \ddots & \\
& & & \sigma_{D}^{2}
\end{array}\right)
$$

Since $\Lambda$ is a diagonal matrix with positive entries, the matrix $\hat{\mathbf{R}} \triangleq\left(1 / \sqrt{N^{2} / 4+N / 2}\right)\left(\mathbf{A}_{\mathbf{1 1}} \boldsymbol{\Lambda} \mathbf{A}_{\mathbf{1 1}}{ }^{H}+\sigma_{n}^{2} \mathbf{I}\right)$ has the same form as the conventional covariance matrix used in subspace based DOA estimation techniques when applied on a ULA with $N^{2} / 4+N / 2$ sensors whose array manifold is represented by $\mathbf{A}_{11}$. It shares the same set of eigenvectors as $\mathbf{R}_{\mathrm{ss}}$ and its eigenvalues are the square roots of those of $\mathbf{R}_{\mathrm{ss}}$. So by eigendecomposition of $\mathbf{R}_{\mathbf{s s}}$, the eigenvectors corresponding to the smallest $N^{2} / 4+N / 2-D$ eigenvalues of $\hat{\mathbf{R}}$ can be found, which will span the null space of $\mathbf{A}_{\mathbf{1 1}}{ }^{H}$ and we can apply subspace based algorithms like MUSIC on it to identify up to $D \leq N^{2} / 4+$ $N / 2-1$ sources.

\section{Remarks}

1) The spatial smoothing technique essentially halves the total degrees of freedom offered by the difference co-array, hence we can identify up to $N^{2} / 4+N / 2-1$ sources as opposed to $N^{2} / 2+N-2$ (if we could exploit total DOF of the full difference co-array). However, using the forward-backward spatial smoothing algorithm, this can be increased to $(3 / 2)\left(N^{2} / 4+N / 2-1\right)$ [25]. The augmented matrix approach [3] or the modified detection estimation algorithm in [5], however, can not identify more than $(1 / 2)\left(N^{2} / 4+N / 2\right)-1$ sources because, as we discussed earlier, they use only one half of the co-array.
2) The co-array of the optimally nested array is not a filled ULA and hence spatial smoothing cannot be used directly on it. It would require some preprocessing like interpolation [24]. However the method based on quasi stationary signals [17] can be applied to it and the full degrees of freedom of the optimally nested array can be exploited as we shall demonstrate through numerical examples.

3) Since $\mathbf{R}_{\mathrm{ss}}$ is constructed as in (12), it is a sum of vector outer products and hence it is positive semidefinite by construction for any finite value of snapshots. This essentially overcomes the major flaw of augmented covariance matrix approach [3] without resorting to the iterative techniques described in [5].

\section{BEAMFORMing With INCREASED DEGREES OF FREEDOM}

As discussed in the last section, the vector $\mathbf{z}$ in (3) can be thought of as the signal received at the difference co-array with the amplitudes of the source signal vector replaced by their corresponding powers. So we can propose beamforming with respect to the signal powers instead of with respect to the signal amplitudes as is the convention. This will enable us to perform beamforming with the full degrees of freedom of the difference co-array and gain $O\left(N^{2}\right)$ degrees of freedom using only $N$ physical sensors. To see this, consider taking the inner product of the vector $\mathbf{z}$ with a weight vector $\mathbf{w}$ to get

$$
y=\mathbf{w}^{H} \mathbf{z}=\sum_{i=1}^{D} \mathbf{w}^{H}\left(\mathbf{a}^{*}\left(\theta_{i}\right) \otimes \mathbf{a}\left(\theta_{i}\right)\right) \sigma_{i}^{2}+\sigma_{n}^{2} \mathbf{w}^{H} \overrightarrow{\mathbf{1}}_{n} .
$$

Defining the new beampattern as

$$
B_{\text {power }}(\theta)=\mathbf{w}^{H}\left(\mathbf{a}^{*}(\theta) \otimes \mathbf{a}(\theta)\right)
$$

we can write (16) as

$$
y=\sum_{i=1}^{D} B_{\text {power }}\left(\theta_{i}\right) \sigma_{i}^{2}+\sigma_{n}^{2} \mathbf{w}^{H} \overrightarrow{\mathbf{1}}_{n} .
$$

Hence, the power of the $i$ th source from direction $\theta_{i}$ gets spatially filtered by the amount $B_{\text {power }}\left(\theta_{i}\right)$ and thereby spatial filtering (or beamforming) is performed with respect to the power of the signal. As is evident, this kind of beamforming is based on a nonlinear preprocessing (taking the autocorrelation of the received signal vector) and hence it is essentially a nonlinear beamformer. It requires time-averaging to realize (2) from which the subsequent signal model is derived. It also requires uncorrelated sources and enough snapshots to realize the autocorrelation matrix. An idea similar to this was discussed in [26] and [27] where beamforming based on time-averaged arrays was suggested using specific array geometries. We now exhibit some examples of different approaches to beamforming that can be performed with this array. Since the two-level nested array has a uniform difference co-array, we would concentrate on beamforming with 2 level nesting since all the conventional beamforming approaches are based on ULAs. However, the beamforming techniques might be extended to the optimally nested array as well. 


\section{A. Deterministic Beamforming}

Let us consider a 2 level nested array with $N / 2$ sensors in each level. The difference co-array is an $U L A$ with $\left(N^{2}-\right.$ $2) / 2+N$ elements. Let $\mathbf{b}(\theta)$ denote the $\left(\left(N^{2}-2\right) / 2+N\right) \times 1$ steering vector of the ULA corresponding to the co-array. Say we want to realize a beam $\mathrm{B}_{\text {des }}(\theta)$ with this ULA and let $\mathbf{w}_{\text {des }}$ denote the deterministic weights such that

$$
\mathbf{w}_{\text {des }}^{H} \mathbf{b}(\theta)=\mathrm{B}_{\text {des }}(\theta) \text {. }
$$

Now to realize this beampattern with the two level nested array, let $\mathbf{w}$ denote the weight vector we would like to apply to the vector $\mathbf{z}$ in (3). Using (19), this means

$$
\mathbf{w}^{H}\left(\mathbf{a}^{*}(\theta) \otimes \mathbf{a}(\theta)\right)=\mathbf{w}_{\text {des }}^{H} \mathbf{b}(\theta), \quad \forall \theta .
$$

From the discussion of the co-array of the 2 level nested array in Section IV, it can be easily seen that the $N^{2} \times 1$ vector $\mathbf{a}^{*}(\theta) \otimes$ $\mathbf{a}(\theta)$ consists of the same rows as $\mathbf{b}(\theta)$, except the fact that some of them occur more than once. Let $n_{i}$ denote the number of times the $i$ th row of $\mathbf{b}(\theta)$ occurs in $\mathbf{a}^{*}(\theta) \otimes \mathbf{a}(\theta)$ and let $i_{1}, i_{2}, \ldots, i_{n_{i}}$ denote the row numbers where it occurs in $\mathbf{a}^{*}(\theta) \otimes \mathbf{a}(\theta)$. Then the weight vector $\mathbf{w}$ is given by

$$
\begin{aligned}
{[\mathbf{w}]_{i_{j}}=\frac{1}{n_{i}}\left[\mathbf{w}_{\text {des }}\right]_{i}, \quad j } & =1,2, \ldots n_{i} \\
i & =1,2, \ldots, \frac{\left(N^{2}-2\right)}{2}+N .
\end{aligned}
$$

\section{B. Nulling of Jammers and Noise}

Consider $J$ jammers incident on the 2 level nested array from directions $\left\{\theta_{i}, i=1,2, \ldots, J\right\}$, respectively. Also assume that we are interested in looking into the direction $\theta_{0}$. Using the proposed beamformer in (19), this means we wish to determine the weight $\mathbf{w}$ to spatially null the jammers and maintain unity response in the look direction. This means

$$
B\left(\theta_{0}\right)=1, \quad B\left(\theta_{i}\right)=0, \quad i=1,2, \ldots, J
$$

However, it can be noticed from (18) that the random noise now assumes the form of a deterministic vector $\overrightarrow{1}_{n}$, which can also be nulled by the weight w. Hence, using this beamforming, we can effectively null the noise term as well. This is something which really cannot be achieved by the conventional beamforming. Thus to null both jammers and noise, w should be selected as the solution to

$$
\left(\begin{array}{c}
\left(\mathbf{a}^{*}\left(\theta_{0}\right) \otimes \mathbf{a}\left(\theta_{0}\right)\right)^{H} \\
\left(\mathbf{a}^{*}\left(\theta_{1}\right) \otimes \mathbf{a}\left(\theta_{1}\right)\right)^{H} \\
\left(\mathbf{a}^{*}\left(\theta_{2}\right) \otimes \mathbf{a}\left(\theta_{2}\right)\right)^{H} \\
\vdots \\
\left(\mathbf{a}^{*}\left(\theta_{J}\right) \otimes \mathbf{a}\left(\theta_{J}\right)\right)^{H} \\
\left(\overrightarrow{\mathbf{1}}_{\mathbf{n}}\right)^{T}
\end{array}\right) \mathbf{w}=\left(\begin{array}{c}
1 \\
0 \\
0 \\
\vdots \\
0 \\
0
\end{array}\right) .
$$

With this beamforming, we can null noise, and up to $\left(N^{2}-\right.$ $2) / 2+N-2$ jammers (with unity response in look direction), since we have in all $\left(N^{2}-2\right) / 2+N$ degrees of freedom in the co-array of the 2-level nested array. The jammer DOAs are either assumed known or can be estimated using the proposed spatial smoothing based method in Section V. This technique for nulling jammers can also be extended to the case of the optimally nested array by simply replacing $\mathbf{a}(\theta)$ of the 2 level nested array with that of the optimally nested one in (21).

It is to be noted that in practice, since the signal covariance matrix is estimated from a finite number of snapshots, the Kronecker product in (21) is only an approximation and hence (21) is satisfied only approximately. However, as we shall show in the numerical examples, the performance is quite satisfactory for moderate number of snapshots.

\section{MVDR-Like Beamforming}

When the jammer directions are not specifically known, MVDR beamforming is a popular alternative where the jammers are automatically suppressed by making the weights inversely proportional to the total covariance matrix of signal+jammer+noise while maintaining a distortionless constraint in the look direction [1]. However the MVDR yields poor performance when the jammers are coherent [29]. In our proposed beamforming, though the incident jammers are originally assumed uncorrelated, after the time averaging process, they are represented by their powers which form a deterministic vector $\mathbf{p}$ in (3). Hence we cannot apply a MVDR directly on $\mathbf{z}$ since the resulting covariance matrix will be of rank 1 . So instead, we perform spatial smoothing on $\mathbf{z}$ as described in the last section by considering the sub-array with elements at $\left\{n d, n=0,1, \ldots, N^{2} / 4+N / 2-1\right\}$ as the reference sub array (whose steering vector we denote as $\mathbf{a}_{1}(\theta)$ ) and get the spatially smoothed covariance matrix $\mathbf{R}_{s s}$ as in (14). This will help us in building a full rank covariance matrix which can be effectively used for MVDR beamforming. With constraint of unity response in the look direction $\theta_{0}$, we can express the MVDR weight as

$$
\mathbf{w}_{M V D R}=\frac{\mathbf{R}_{s s}^{-1} \mathbf{a}_{1}\left(\theta_{0}\right)}{\mathbf{a}_{1}\left(\theta_{0}\right)^{H} \mathbf{R}_{s s}^{-1} \mathbf{a}_{1}\left(\theta_{0}\right)} .
$$

\section{NUMERICAL EXAMPLES}

In this section, we provide numerical examples to illustrate the superior performance of the nested array in terms of degrees of freedom for DOA estimation and beamforming. In the examples, we consider a 6 sensor array $(N=6)$ with 8 narrowband sources $(D=8)$ impinging on it from directions of arrival $\left\{-60^{\circ},-45^{\circ},-30^{\circ}, 0^{\circ}, 15^{\circ}, 30^{\circ}, 45^{\circ}, 60^{\circ}\right\}$, all with equal power. The sources are modeled as random Gaussian processes. For comparing with the KR product based MUSIC [17] which requires quasi stationarity, we assume 16 different quasi stationary intervals with $T_{1}$ snapshots in each interval. The noise is assumed to be spatially and temporally white. For being able to apply spatial smoothing, we consider a 2 level nested array with 3 sensors in each level. The method based on spatial smoothing can resolve up to $N^{2} / 4+N / 2-1=11$ sources. The KR-product based method can resolve $\left(N^{2}-2\right) / 2+N-1=22$ sources but requires quasi-stationary signals and a larger number of snapshots compared to the proposed method. In particular, if the number 


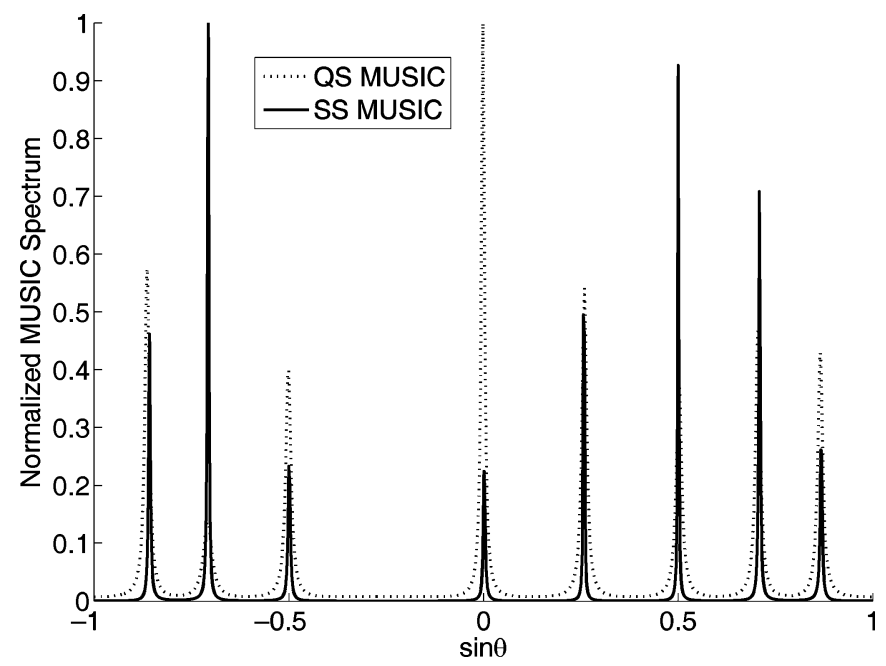

Fig. 3. MUSIC spectrum using the SS-method and the QS-method, as a function of sine of the DOA, $N=6, D=8, T=4800, \mathrm{SNR}=0 \mathrm{~dB}$.

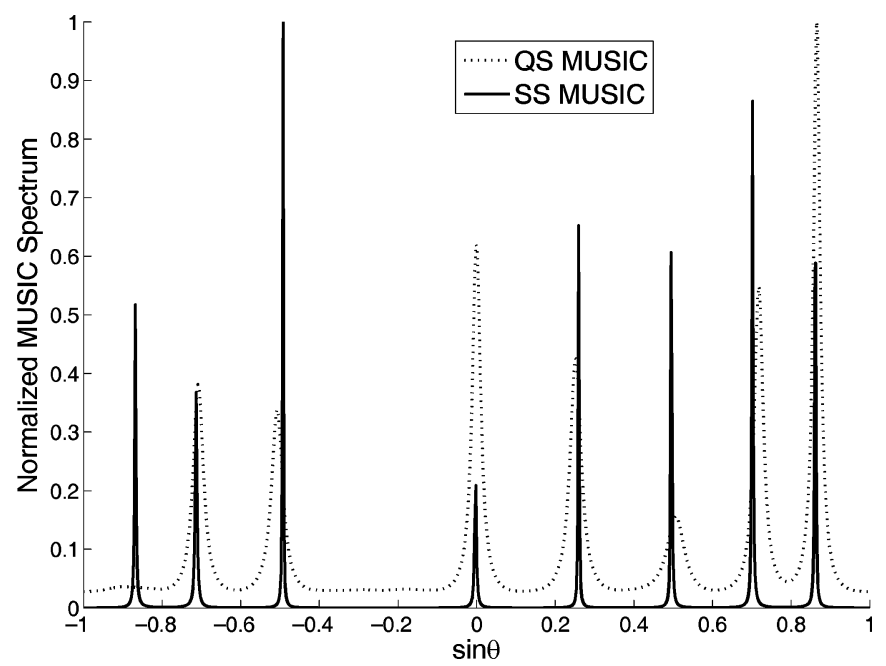

Fig. 4. MUSIC spectrum using the SS-method and QS-method, as a function of sine of the DOA. Here $N=6, D=8, T=480$, SNR $=0 \mathrm{~dB}$.

of sources is $D$, then the KR-product based method requires at least $D$ times more snapshots than the proposed spatial smoothing based method.

\section{A. Music Spectra}

Fig. 3 shows the representative MUSIC spectra after applying the proposed spatial smoothing based technique (SS-method) and the quasi stationary based techniques (QS-method). Both use a total of $T=4800$ snapshots with the KR product based MUSIC using $T_{1}=300$ snapshots for each of the 16 quasistationary intervals. The SNR for both the methods is assumed to be $0 \mathrm{~dB}$. As can be seen, both of them can resolve the 8 sources sufficiently well.

Now let us reduce the total number of snapshots to $T=$ 480 snapshots. Fig. 4 illustrates the MUSIC spectra for the proposed SS-method and the QS-method. The QS method clearly suffers due to lack of snapshots and it misses the peak at $-60^{\circ}$ whereas the SS method still shows a good performance with clearly discernible peaks of the MUSIC spectrum. This shows

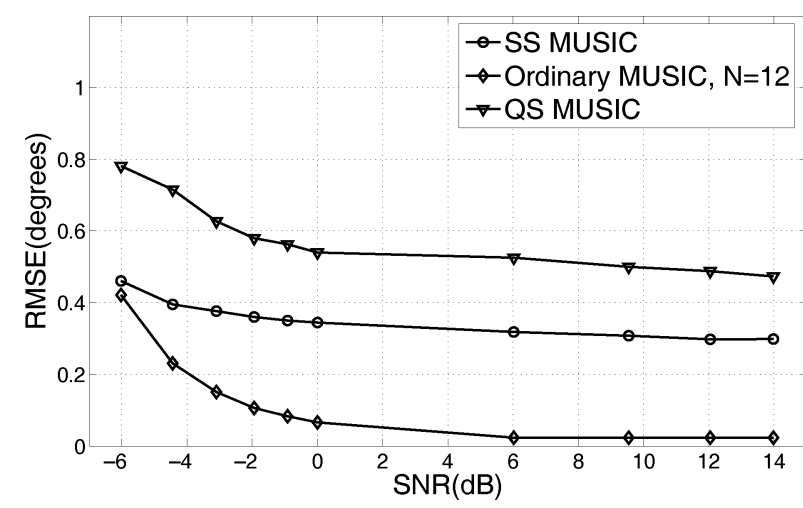

Fig. 5. RMSE (in degrees) versus SNR (for the source at $30^{\circ}$ ) of QS and SS methods applied on 2 level nested array and ordinary MUSIC on a 12 element ULA, with $T=800, N=6$.

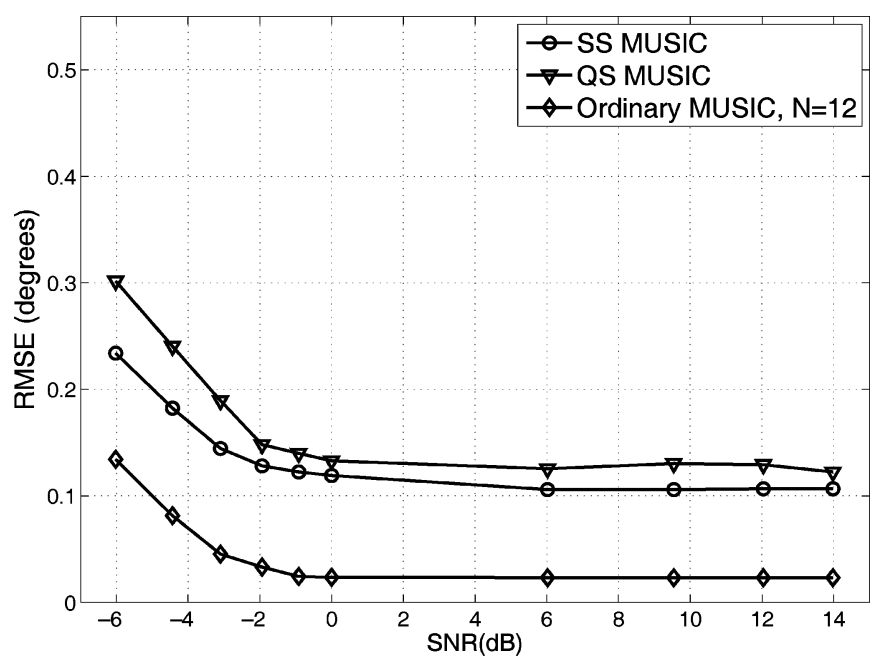

Fig. 6. RMSE (in degrees) versus SNR (for the source at $30^{\circ}$ ) of QS and SS methods applied on 2 level nested array and ordinary MUSIC on a 12 element ULA, with $T=4800, N=6$.

the effect of snapshots on the performance of the two methods. Next, we study the performance of both the methods through Monte Carlo simulations.

\section{B. RMSE versus SNR and Snapshots}

We compare the SS-method and QS-methods by studying the RMSE of the angle estimates as a function of both SNR and snapshots. Since the two level array has 12 degrees of freedom, we also consider the corresponding RMSE for conventional MUSIC applied to a 12 element ULA which we would use as a benchmark at high SNR. We plot the RMSE for the source at $30^{\circ}$. The performance is similar for the other sources as well. Fig. 5 shows the RMSE of the three methods as a function of SNR, averaged over 1500 Monte Carlo simulations, for $T=800$ snapshots. Fig. 6 show the corresponding performances for $T=4800$ snapshots.

Notice how the performance of both SS and QS methods improve with SNR. Also, the SS method performs reasonably better than the KR method at low snapshots whereas the difference in their performance becomes less prominent at larger number of snaps. Note, both the methods perform worse than the conventional MUSIC applied to a 12 element ULA because 


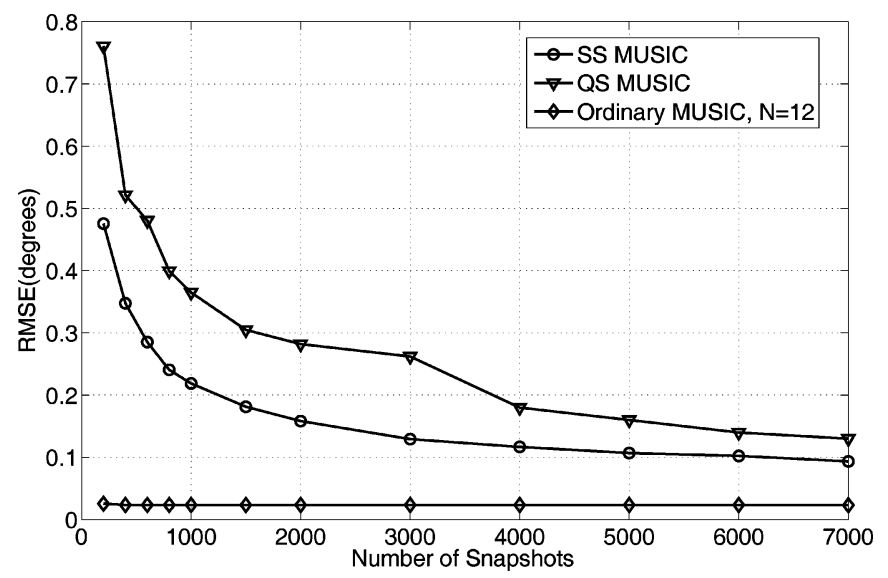

Fig. 7. RMSE (in degrees) versus the number of snapshots $T$ (for the source at $30^{\circ}$ ) of QS and SS methods applied on 2 level nested array and ordinary MUSIC on a 12 element ULA. Here SNR $=6 \mathrm{~dB}, N=6$.

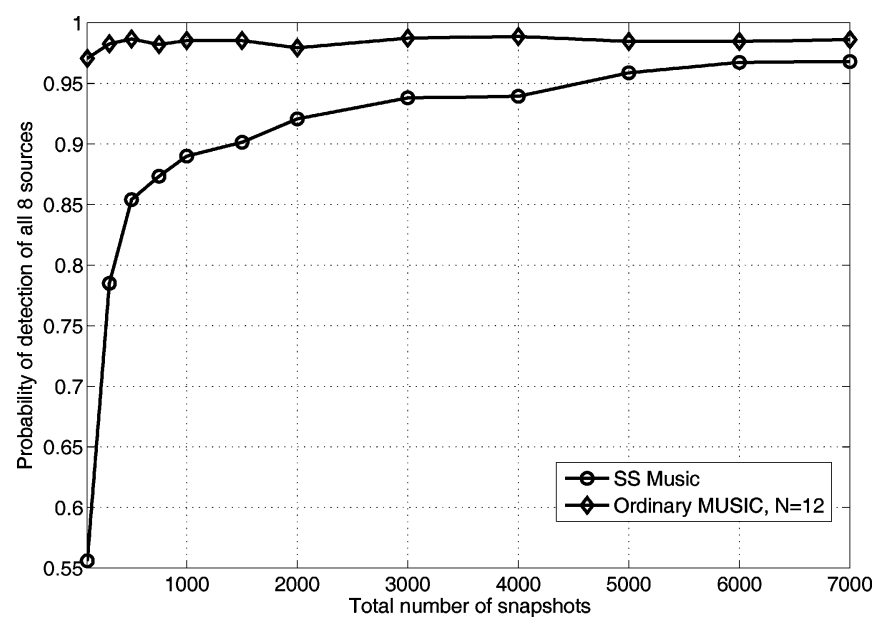

Fig. 8. Comparison of detection performance of SS method applied to 2 level nested array and MUSIC applied to 12 element ULA as a function of number of snapshots. Here SNR $=10 \mathrm{~dB}, N=6$.

of the finite sample size due to which the kronecker product in (17) is only an approximation.

Next, in Fig. 7 we plot the performance of the three methods for SNR $=6 \mathrm{~dB}$ by varying the total number of snapshots $T$. The performance of both SS and QS improve considerably with the number of snapshots with the QS method performing strictly worse than the proposed SS method.

\section{Detection Performance}

In the previous examples, we assumed the number of source $D$ to be known. However, in practical situations, we need to estimate it from the available data. To do this, we would need to find some optimum detection algorithm suited to our model, which is a subject of our future research in this direction. However, we applied the threshold based technique in [30] which proved to give satisfactory results in the proposed SS based technique. We plot the estimated probability of detection of this proposed method at $\mathrm{SNR}=10 \mathrm{~dB}$ as a function of snapshots in Fig. 8. For comparison, we also plot the corresponding performance of the conventional MUSIC applied on 12 element ULA. The probabilities were obtained by running 1000 Monte Carlo simulations

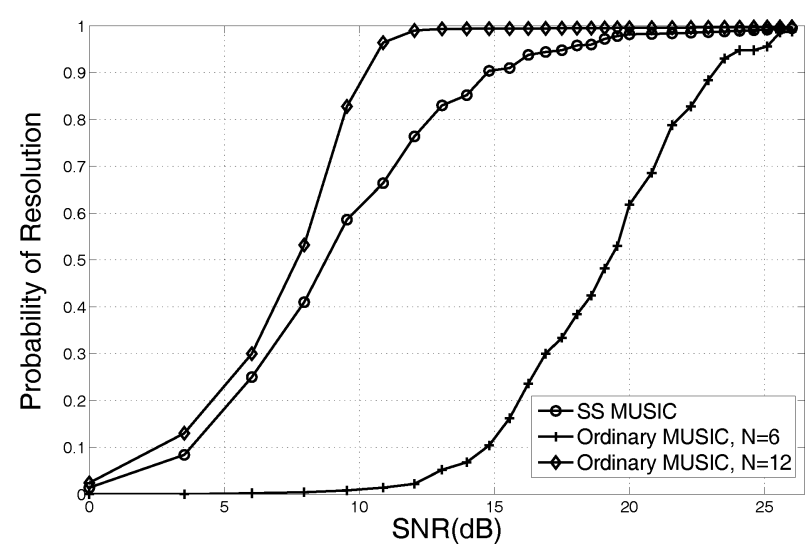

Fig. 9. Comparison of resolution performance of SS-MUSIC method applied to 2 level nested array with 6 sensors and traditional MUSIC applied to a 12 element ULA and a 6 element ULA, as a function of SNR for two closely spaced sources at $12^{\circ}$ and $14^{\circ}$.

averaged over another 1000 runs. We can see that the detection performance improves dramatically with increasing snapshots and approaches that of the conventional MUSIC asymptotically.

\section{Resolution Performance}

The advantage of MUSIC-like algorithms comes from the fact that these are super-resolution algorithms, capable of resolving even very closely spaced sources. Since SS-MUSIC makes use of the increased degrees of freedom provided by the co-array, we expect its resolution performance to improve greatly. In this example, we consider two sources placed at $12^{\circ}$ and $14^{\circ}$ and compare the resolution performances of two-level nested array with 6 sensors, an ULA with 6 sensors and an ULA with 12 sensors (since SS MUSIC applied on 6 element ULA yields 12 degrees of freedom). Following [31], we plot the probability of resolution versus SNR for $T=300$ snapshots, averaged over 1000 Monte Carlo runs in Fig. 9. As in [31], we assume that the number of sources is known. Clearly the two level nested array outperforms the corresponding ULA with same number of sensors and performs close to the much longer ULA.

\section{E. 2 Level versus Optimally Nested Array}

We now provide an example to show the ability of the optimally nested array to resolve more sources than the corresponding 2 level array for $N=6$ sensors. Since the spatial smoothing based technique cannot be applied directly to the optimally nested array, we consider QS method to perform the DOA estimation. With QS method, the 2 level array can resolve $D=22$ sources whereas the optimally nested array can resolve 30 sources. We consider 27 quasi-stationary sources distributed uniformly between $-60^{\circ}$ and $60^{\circ}$. We use $2 \times 27=54$ quasi-stationary intervals with $T_{1}=300$ snapshots per interval yielding a total of $54 \times 300=16200$ snapshots. Figs. 10 and 11 , respectively, show the corresponding spectrum obtained by using QS-MUSIC to the optimally nested array and the 2 level nested array. Clearly, the 2 level nested array fails to detect the sources whereas the optimally nested array can perfectly detect 


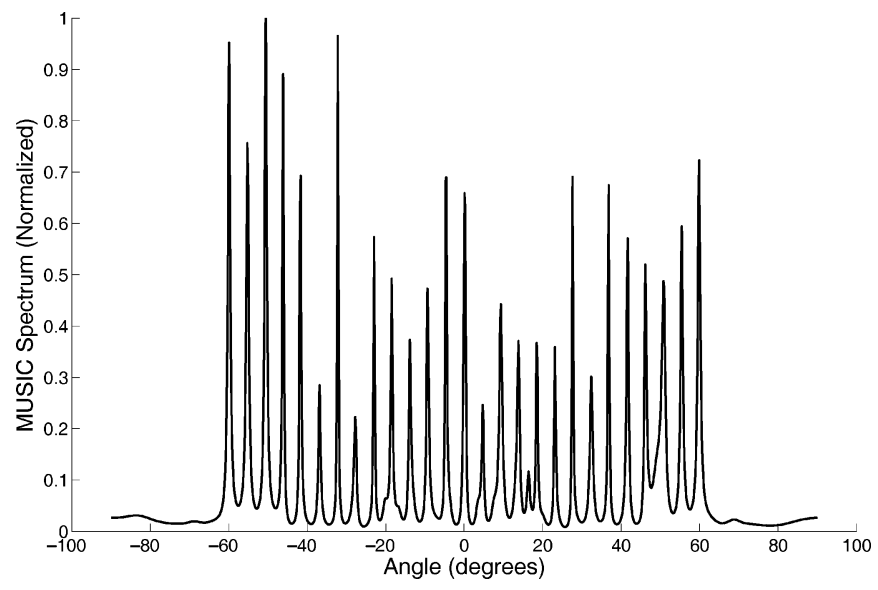

Fig. 10. MUSIC spectrum for QS-method applied to the optimally nested array with $N=6, T=16200, D=27$ and $\mathrm{SNR}=0 \mathrm{~dB}$.

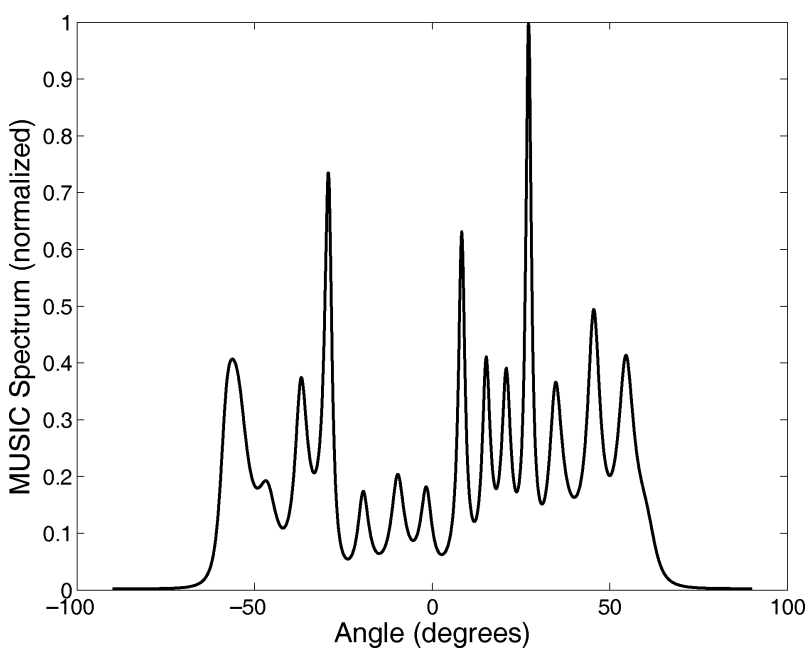

Fig. 11. MUSIC spectrum for QS-method applied to the 2 level nested array with $N=6, T=16200, D=27$, and SNR $=0 \mathrm{~dB}$.

them. This confirms the superior detection ability of the optimally nested array. For large $N$, the optimally nested array can detect about twice as many sources as the two-level nested array.

\section{F. Beamforming}

In this section, we demonstrate the capability to perform beamforming with increased degrees of freedom offered by the 2 level nested array. There are two ways of plotting the resultant beampattern. One way is to plot the pattern $B(\theta)$ as defined in (17) directly after computing the necessary weights. This, however, is not practical because the kronecker product in (17) is realized only approximately due to finite snapshots. Hence, for practical reasons, to plot a more realistic beampattern, we consider a single source at angle $\theta$ and plot the amplitude response of our nonlinear beamformer to it by varying $\theta$ for finite number of snapshots. This automatically takes into consideration the finite sample effect. We call the former beam as an desired beampattern and the latter as the practical beampattern.

1) Deterministic Beampattern: Let us consider a simple sinc beampattern obtained by applying uniform weighting to a $19 \mathrm{el}-$ ement array. Since $N=6$ element array with 2 level of nesting

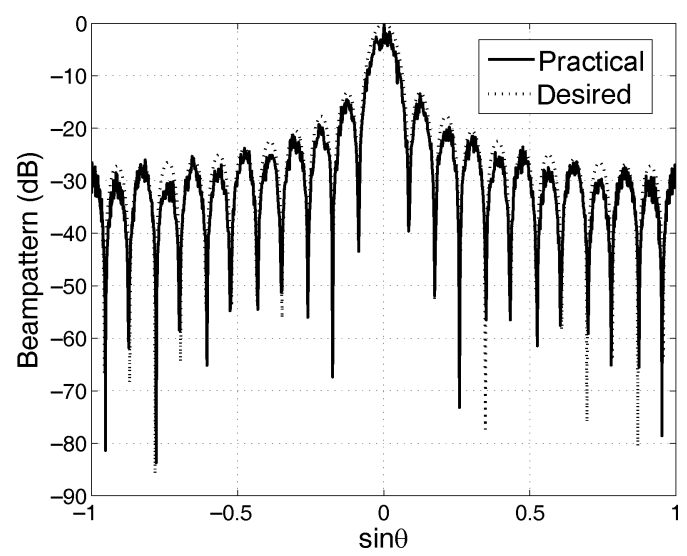

Fig. 12. Practical deterministic sinc beampattern obtained by nonlinear beamforming from the 2 level nested array, $N=6, T=100$.

gives $\left(N^{2}-2\right) / 2+N=23$ degrees of freedom, we consider a 6 element array and plot both the desired and practical beampatterns in Fig. 12 by computing the weights following (20). The practical beampattern is generated using $T=100$ snapshots and it approximates the ideal beampattern quite closely.

2) Jammer and Noise Nulling: Consider a 4 element array with 2 levels of nesting. The look direction is $0^{\circ}$ and we consider 6 jammers from directions $\left\{-60^{\circ},-45^{\circ},-30^{\circ}, 15^{\circ}, 30^{\circ}, 45^{\circ}\right\}$ with jammer to signal power ratio $20 \mathrm{~dB}$ and signal to noise ratio $0 \mathrm{~dB}$. The desired and practical beampatterns are generated using the weight computed from (21) and plotted in Fig. 14. Note the introduction of nulls along the jammer positions. Also, the sidelobes are seen to be high which could pose problems for noise in the conventional beamforming. However, unlike conventional beamforming, in our case the noise has been reduced to a deterministic vector which can also be efficiently nulled. So the presence of noise at all spatial angles (which is assumed in conventional beamforming) can be ruled out and, hence, the high sidelobes do not pose a serious threat as far as noise is concerned. Also, we see that though this jammer and noise nulling theoretically requires perfect knowledge of signal covariance matrix, the practical beampattern shows that the approximation is reasonably good at moderate number of snapshots $(T=100)$.

In order to show the performance improvement of our proposed beamforming, we plot the signal to jammer plus noise ratio (SJNR) versus the number of snapshots in Fig. 13. The SJNR plot is obtained as follows. The response of a beamformer to an input signal consisting of desired signal+jammer+noise is measured. Then the response of the beamformer to the input signal consisting only of the same jammer+noise is measured. Finally the ratio between the difference of the first and second responses, and the second response is taken to be the SJNR and it is plotted on a dB scale, averaged over 1000 Monte Carlo simulations. Notice how the SJNR performance of proposed method improves with number of snapshots. We also compare it with the SJNR performance of an equivalent ULA with 11 sensors (since the co-array of a 2 level nested array with 4 sensors has 11 degrees of freedom) where we introduced nulls along jammer directions and used remaining degrees of freedom to maximize the SNR. As expected, the SJNR of the equivalent ULA is not affected by number of snapshots. Also, it is to be 


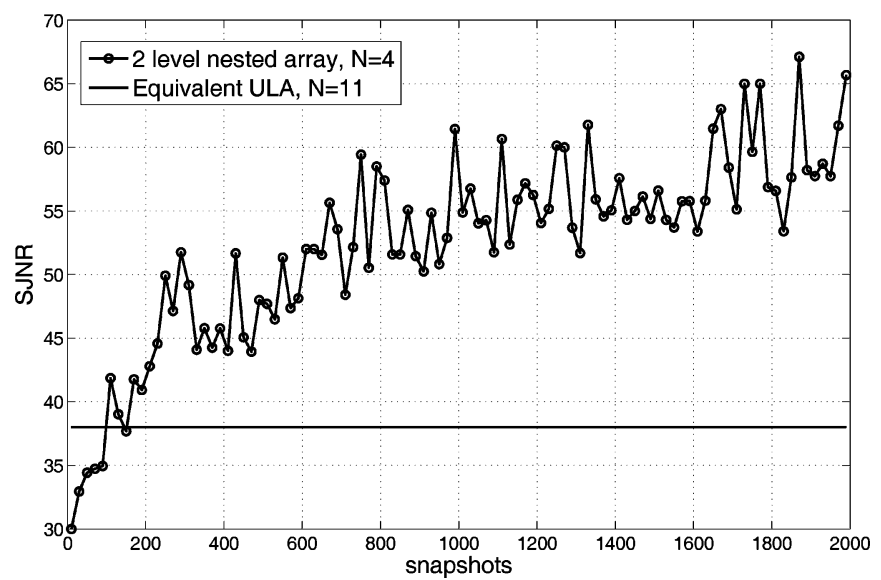

Fig. 13. Signal-to-Jammer-and-noise-ratio (SJNR) versus number of snapshots (averaged over 1000 Monte Carlo runs) for 2 level nested array with four sensors and the equivalent ULA with 11 sensors, Number of jammers $=6, \mathrm{SNR}=$ $0 \mathrm{~dB}, \mathrm{SJR}=-20 \mathrm{~dB}$.

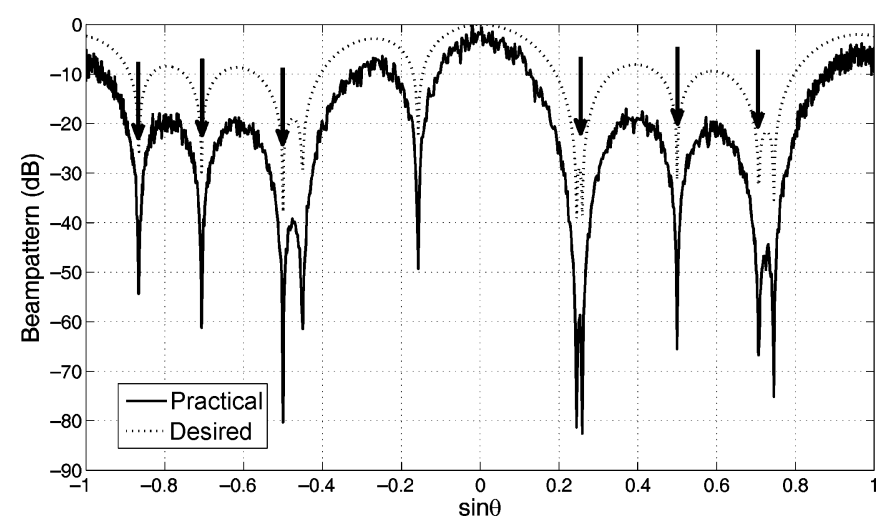

Fig. 14. Practical Beampattern obtained from the 2 level nested array after nulling 6 jammers and noise, $N=4, T=100$.

noted that the proposed beamforming outperforms the ULA. This can be explained from the fact that we are actually able to null the noise (the extent to which it is nulled again depends on snapshots) which is not possible for the ordinary beamforming case with the equivalent ULA, and hence the SJNR for our method increases almost linearly (on $\mathrm{dB}$ scale) with the number of snapshots.

3) MVDR-Like Beampattern: We now plot the MVDR-like beampattern for the spatially smoothed 6 element array with 2 levels of nesting. Look direction is $0^{\circ}$ and we consider 9 jammers at $\left\{-60^{\circ},-45^{\circ},-30^{\circ},-15^{\circ}, 20^{\circ}, 30^{\circ}, 45^{\circ}\right.$, $55^{\circ}, 60^{\circ}$ \}. The weights are computed as per (22) using $T=$ 1000 snapshots to compute the spatially smoothed covariance matrix $\mathbf{R}_{s s}$, and the desired beampattern is plotted in Fig. 15.

Through the inversion of the spatially smoothed matrix in (22), nulls have been automatically introduced in the jammer directions even without explicitly finding the jammer DOAs.

\section{CONCLUSION}

In this paper, we have attempted a unified viewpoint to compare the established approaches to the problem of underdetermined source localization in the light of the co-array concept. Designing arrays for high resolution array processing was also addressed in [33]. Drawing inspiration from the MIMO radar

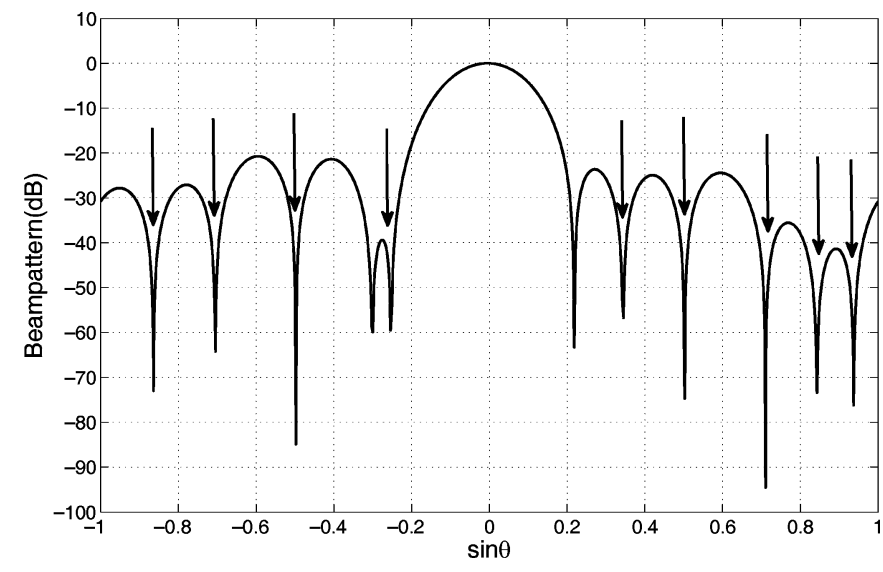

Fig. 15. Desired MVDR-like beampattern obtained by applying spatial smoothing to the 2 level nested array, $N=6$, using $T=1000$ snapshots for computing the smoothed covariance matrix.

literature, we proposed a novel nested array structure which can realize significantly more degrees of freedom even in the passive sensing scenario. Different nesting strategies were explored and the optimum nested array structure was found through solving a combinatorial optimization problem. Though this nested array could be used in conjunction with both the fourth-order cumulant based and the KR-product based methods, it would suffer from the inherent drawbacks of these techniques. So we proposed an alternative spatial smoothing based approach to underdetermined DOA estimation, which does not require any of the assumptions in the previous two methods. We also demonstrated how to perform beamforming with this array structure. The results were verified through simulations and the performances of the proposed techniques are seen to improve considerably by increasing the number of snapshots.

Future research in this area will be directed towards finding an optimum detection algorithm for the proposed nested array structure, deducing the optimum beamforming policy to maximize signal to jammer and noise ratio and also extension of these ideas to the case of wideband signals. Another important direction would be to evaluate the performance of the proposed method (based on the construction of the spatially smoothed matrix) analytically and derive theoretical insights into behavior of the proposed algorithms for finite snapshots, SNR and number of impinging sources.

\section{APPENDIX \\ PROOF OF THEOREM 1}

Using the constraint in (10), we can write (9) as

$$
\begin{aligned}
\operatorname{DOF}_{K} & =2\left\{\frac{1}{2}\left[\left(\sum_{i=1}^{K} N_{i}\right)^{2}-\sum_{i=1}^{K} N_{i}^{2}\right]+N_{K}\right\}-1 \\
& =N^{2}-\sum_{i=1}^{K} N_{i}^{2}+2 N_{K}-1 .
\end{aligned}
$$

Let us consider $N_{j}$, the number of sensors in the $j$ th level of nesting. We shall deal with the following two cases separately:

- Case I: $1 \leq j \leq K-1$. Consider breaking $N_{j}$ into the sum of two smaller integers $N_{j 1}$ and $N_{j 2}$, i.e., $N_{j}=N_{j 1}+N_{j 2}$. This amounts to breaking the $j$ th nested level into 2 levels 
such that now there are $(K+1)$ levels of nesting with $\left\{N_{1}, N_{2}, \ldots, N_{j-1}, N_{j 1}, N_{j 2}, N_{j+1}, \ldots, N_{K}\right\}$ sensors, respectively. Using the expression for degrees of freedom in (23), we can write, the new degrees of freedom for this new nested array, as

$\operatorname{DOF}_{K+1}=N^{2}-\left[\sum_{i \neq j, i=1}^{K} N_{i}^{2}+N_{j 1}^{2}+N_{j 2}^{2}\right]+2 N_{K}-1$.

Subtracting (23) from (24), we get the change in DOF due to splitting the $j$ th nesting level:

$$
\begin{aligned}
\triangle \mathrm{DOF} \triangleq \mathrm{DOF}_{K+1}-\mathrm{DOF}_{K} & =N_{j}^{2}-N_{j 1}^{2}-N_{j 2}^{2} \\
& =2 N_{j 1} N_{j 2}>0 .
\end{aligned}
$$

Thus we can conclude that breaking up the jth level of nesting into two levels, always increases the degrees of freedom for $j=1,2,3, \ldots, K-1$. Hence we should go on redistributing the sensors in the inner nesting levels till we hit only one sensor per nesting level. So, at this stage we have $\hat{N}_{K}$ elements in the last stage and all the stages before it have one sensor each, i.e., $N_{i}=1, \quad i=1,2,3 \ldots, K-$ 1 Now we consider the last stage of nesting and optimize the number of sensors in them.

- Case II: $j=K$. Similar to the previous case, let us break $N_{K}$ as $N_{K}=N_{K 1}+N_{K 2}$ So now we have a $K+1$ level-nested array, with $N_{1}, N_{2}, \ldots, N_{K 1}, N_{K 2}$ sensors, respectively, in the $K+1$ levels. Applying the formula for degrees of freedom in (23), we get the degrees of freedom in this case as

$\mathrm{DOF}_{K+1, K}=N^{2}-\left[\sum_{i=1}^{K-1} N_{i}^{2}+N_{K 1}^{2}+N_{K 2}^{2}\right]+2 N_{K 2}-1$.

Similarly as before, the increase in degrees of freedom can be found by subtracting (23) from (25) as

$$
\begin{aligned}
\triangle \mathrm{DOF}_{K+1, K} & \triangleq \mathrm{DOF}_{K+1, K}-\mathrm{DOF}_{K} \\
& =N_{K}^{2}-N_{K 1}^{2}-N_{K 2}^{2}+2\left(N_{K 2}-N_{K}\right) \\
& =2 N_{K 1} N_{K 2}-2 N_{K 1} \\
& =2 N_{K 1}\left(N_{K 2}-1\right) \geq 0 .
\end{aligned}
$$

Thus we see that in this case also, the degrees of freedom increase by splitting as long as the number of sensors in the last level (i.e., $N_{K 2}$ ) is $\geq 2$. So we should go on splitting the last level of nesting till we hit 2 sensors in the last level and one sensor in every other level. This, along with the previous case, also implies that the total number of levels of nesting is $N-2+1=N-1$ since the total number of sensors is $N$.

\section{REFERENCES}

[1] H. L. Van Trees, Optimum Array Processing: Part IV of Detection, Estimation and Modulation Theory. New York: Wiley Intersci., 2002.

[2] R. Schmidt, "Multiple emitter location and signal parameter estimation," IEEE Trans. Antennas Propag., vol. 34, pp. 276-280, Mar. 1986.
[3] S. U. Pillai, Y. Bar-Ness, and F. Haber, "A new approach to array geometry for improved spatial spectrum estimation," Proc. IEEE, vol. 73, pp. 1522-1524, Oct. 1985.

[4] S. Pillai and F. Haber, "Statistical analysis of a high resolution spatial spectrum estimator utilizing an augmented covariance matrix," IEEE Trans. Acoust., Speech Signal Process., vol. 35, no. 11, pp. 1517-1523, Nov. 1987.

[5] Y. I. Abramovich, D. A. Gray, A. Y. Gorokhov, and N. K. Spencer, "Positive-definite Toeplitz completion in DOA estimation for nonuniform linear antenna arrays. I. Fully augmentable arrays," IEEE Trans. Signal Process., vol. 46, pp. 2458-2471, Sep. 1998.

[6] Y. I. Abramovich, N. K. Spencer, and A. Y. Gorokhov, "Positive-definite Toeplitz completion in DOA estimation for nonuniform linear antenna arrays. II. Partially augmentable arrays," IEEE Trans. Signal Process., vol. 47, pp. 1502-1521, Jun. 1999.

[7] D. H. Johnson and D. E. Dudgeon, Array Signal Processing - Concepts and Techniques. Englewood Cliffs, NJ: Prentice-Hall, 1993.

[8] D. A. Linebarger, I. H. Sudborough, and I. G. Tollis, "Difference bases and sparse sensor arrays," IEEE Trans. Inf. Theory, vol. 39, pp. 716-721, Mar. 1993.

[9] A. Moffet, "Minimum-redundancy linear arrays," IEEE Trans. Antennas Propag., vol. 16, pp. 172-175, Mar. 1968.

[10] C.-Y. Chen and P. P. Vaidyanathan, "Minimum redundancy MIMO radars," in Proc. IEEE Int. Symp. Circuits Syst. (ISCAS), 2008, May 18-21, 2008, pp. 45-48.

[11] D. Pearson, S. U. Pillai, and Y. Lee, "An algorithm for near-optimal placement of sensor elements," IEEE Trans. Inf. Theory, vol. 36, pp. 1280-1284, Nov. 1990.

[12] C. S. Ruf, "Numerical annealing of low-redundancy linear arrays," IEEE Trans. Antennas Propag., vol. 41, pp. 85-90, Jan. 1993.

[13] B. Porat and B. Friedlander, "Direction finding algorithms based on high-order statistics," IEEE Trans. Signal Process., vol. 39, pp. 2016-2024, Sep. 1991.

[14] M. C. Dogan and J. M. Mendel, "Applications of cumulants to array processing .I. Aperture extension and array calibration," IEEE Trans. Signal Process., vol. 43, pp. 1200-1216, May 1995.

[15] P. Chevalier, L. Albera, A. Ferreol, and P. Comon, "On the virtual array concept for higher order array processing," IEEE Trans. Signal Process., vol. 53, pp. 1254-1271, Apr. 2005.

[16] P. Chevalier and A. Ferreol, "On the virtual array concept for the fourth-order direction finding problem," IEEE Trans. Signal Process., vol. 47, pp. 2592-2595, Sep. 1999.

[17] W.-K. Ma, T.-H. Hsieh, and C.-Y. Chi, "DOA estimation of quasistationary signals via Khatri-Rao subspace," in Proc. Int. Conf. Acoust., Speech Signal Process. (ICASSP), Apr. 2009, pp. 2165-2168.

[18] D. W. Bliss and K. W. Forsythe, "Multiple-input multiple-output (MIMO) radar and imaging: Degrees of freedom and resolution," in Proc. 37th IEEE Asilomar Conf. Signals, Syst., Comput., Nov. 2003, vol. 1, pp. 54-59.

[19] R. T. Hoctor and S. A. Kassam, "The unifying role of the coarray in aperture synthesis for coherent and incoherent imaging," Proc. IEEE, vol. 78, pp. 735-752, Apr. 1990.

[20] R. J. Kozick and S. A. Kassam, "Coarray synthesis with circular and elliptical boundary arrays," IEEE Trans. Image Process., vol. 1, pp. 391-405, Jul. 1992.

[21] C.-Y. Chen and P. P. Vaidyanathan, "MIMO radar space-time adaptive processing using prolate spheroidal wave functions," IEEE Trans. Signal Process., vol. 56, pp. 623-635, Feb. 2008.

[22] E. Evans, J. R. Johnson, and D. F. Sun, "High resolution angular spectrum estimation techniques for terrain scattering analysis and angle of arrival estimation," in Proc. 1st ASSP Workshop Spectral Estimation, Hamilton, Ontario, Canada, Aug. 1981, pp. 134-139.

[23] T.-J. Shan, M. Wax, and T. Kailath, "On spatial smoothing for direction-of-arrival estimation of coherent signals," IEEE Trans. Acoust., Speech Signal Process., vol. 33, no. 4, pp. 806-811, Aug. 1985.

[24] B. Friedlander and A. J. Weiss, "Direction finding using spatial smoothing with interpolated arrays," IEEE Trans. Aerosp. Electron. Syst., vol. 28, pp. 574-587, Apr. 1992.

[25] S. U. Pillai and B. H. Kwon, "Forward/backward spatial smoothing techniques for coherent signal identification," IEEE Trans. Acoust., Speech Signal Process., vol. 37, no. 1, pp. 8-15, Jan. 1989.

[26] H.P. Bucker, "High resolution cross-sensor beamforming for a billboard array," J. Acoust. Soc. Amer., vol. 65, no. 1, pp. 145-147, Jan. 1979.

[27] H. P. Bucker, "Cross-sensor beam forming with a sparse line array," $J$. Acoust. Soc. Amer., vol. 61, no. 2, pp. 494-498, Feb. 1977.

[28] H. C. Pumphrey, "Design of sparse arrays in one, two and three dimensions," J. Acoust. Soc. Amer., vol. 93, no. 3, pp. 1620-1628, Mar. 1993. 
[29] M. D. Zoltowski, "On the performance analysis of the MVDR beamformer in the presence of correlated interference," IEEE Trans. Acoust., Speech Signal Process., vol. 36, no. 6, pp. 945-947, Jun. 1988.

[30] W. Chen, K. M. Wong, and J. P. Reilly, "Detection of the number of signals - A predicted eigen-threshold approach," IEEE Trans. Signal Process., vol. 39, pp. 1088-1098, May 1991.

[31] M. Kaveh and A. Barabell, "The statistical performance of the MUSIC and the minimum-norm algorithms in resolving plane waves in noise," IEEE Trans. Acoust., Speech Signal Process., vol. 34, no. 2, pp. 331-341, Apr. 1986.

[32] H. Krim and M. Viberg, "Two decades of array signal processing research: The parametric approach," IEEE Signal Process. Mag., vol. 13, pp. 67-94, Jul. 1996.

[33] S. W. Lang, G. L. Duckworth, and J. H. McClellan, "Array design for MEM and MLM array processing," in Proc. Int. Conf. Acoustics, Speech, Signal Procesing (ICASSP), 1981, pp. 145-148.

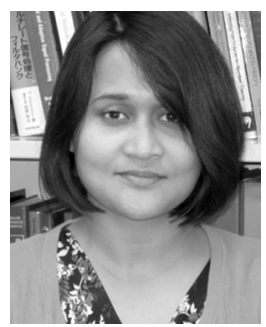

Piya Pal (S'08) was born in Calcutta, India, on September 30, 1984. She received the B.Tech. degree in electronics and electrical communication engineering from the Indian Institute of Technology, Kharagpur, in 2007, and the M.S. degree in electrical engineering from the California Institute of Technology (Caltech), Pasadena, in 2008.

She is currently pursuing the Ph.D. degree in the field of digital signal processing at Caltech. Her research interests are in digital signal processing and applications in sensor and array signal processing.

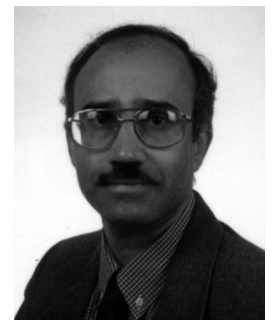

P. P. Vaidyanathan (S'80-M'83-SM'88-F'91) was born in Calcutta, India, on October 16, 1954 He received the B.Sc. (Hons.) degree in physics and the B.Tech. and M.Tech. degrees in radiophysics and electronics, all from the University of Calcutta, in 1974, 1977, and 1979, respectively, and the Ph.D. degree in electrical and computer engineering from the University of California, Santa Barbara (UCSB) in 1982.

He was a Postdoctoral Fellow with the UCSB from September 1982 to March 1983. In March 1983, he joined the Electrical Engineering Department, California Institute of Technology (Caltech), Pasadena, as an Assistant Professor, and since 1993 , has been a Professor of Electrical Engineering. His main research interests are in digital signal processing, multirate systems, wavelet transforms, signal processing for digital communications, genomic signal processing, radar signal processing, and sparse array signal processing. He has authored more than 400 papers in journals and conferences, and is the author of the three books Multirate Systems and Filter Banks (Englewood Cliffs: NJ Prentice-Hall, 1993), Linear Prediction Theory (Morgan and Claypool, 2008), and (with Phoong and Lin) Signal Processing and Optimization for Transceiver Systems (Cambridge, U.K.: Cambridge University Press, 2010). He has written several chapters for various signal processing handbooks.

Dr. Vaidyanathan served as Vice-Chairman of the Technical Program committee for the 1983 IEEE International symposium on Circuits and Systems, and as the Technical Program Chairman for the 1992 IEEE International symposium on Circuits and Systems. He was an Associate Editor for the IEEE TRANSACTIONS ON CiRCUITS AND SySTEMS during 1985-1987, and is currently an Associate Editor for the IEEE SIGNAL PROCESSING LETTERS, and a consulting editor for Applied and Computational Harmonic Analysis. He has been a Guest Editor in 1998 for Special Issues of the IEEE TRANSACTIONS ON Signal Processing and the IEEE TRANSACtions ON CIRCUITS AND SYSTEMS II, on the topics of filter banks, wavelets, and subband coders. He was a recipient of the Award for Excellence In Teaching at Caltech for 1983-1984, 1992-1993, and 1993-1994. He also received the NSF's Presidential Young Investigator award in 1986. In 1989, he received the IEEE ASSP Senior Award for his paper on multirate perfect-reconstruction filter banks. In 1990, he was the recipient of the S. K. Mitra Memorial Award from the Institute of Electronics and Telecommunications Engineers, India, for his joint paper in the IETE journal. In 2009, he was chosen to receive the IETE students' journal award for his tutorial paper in the IETE Journal of Education. He was also the coauthor of a paper on linear-phase perfect reconstruction filter banks in the IEEE TRANSACTIONS ON Signal PROCESSING, for which the first author (T. Nguyen) received the Young Outstanding Author award in 1993. He received the 1995 F. E. Terman Award of the American Society for Engineering Education, sponsored by Hewlett Packard Co., for his contributions to engineering education. He has given several plenary talks including at the IEEE ISCAS-04, Sampta-01, Eusipco-98, SPCOM-95, and Asilomar-88 conferences on signal processing. He has been chosen a Distinguished Lecturer for the IEEE Signal Processing Society for the year 1996-1997. In 1999, he was chosen to receive the IEEE CAS Society's Golden Jubilee Medal. He is a recipient of the IEEE Signal Processing Society's Technical Achievement Award for 2002. 